KAPL-P-000148

(K97011)

\title{
NEUTRON DETECTOR RESOLUTION FOR SCATTERING
}

S. A. Kolda, D. Mesh

March 1997

DISTAIBUTION OF THIS DOCUMENT IS UNLIMTED \&

\section{NOTICE}

This report was prepared as an account of work sponsored by the United States Government. Neither the United States, nor the United States Department of Energy, nor any of their employees, nor any of their contractors, subcontractors, or their employees, makes any warranty, express or implied, or assumes any legal liability or responsibility for the accuracy, completeness or usefulness of any information, apparatus, product or process disclosed, or represents that its use would not infringe privately owned rights. 


\section{DISCLAIMER}

This report was prepared as an account of work sponsored by an agency of the United States Government. Neither the United States Government not any agency thereof, nor any of their employees, makes any warranty, express or implied, or assumes any legal liability or responsibility for the accuracy, completereas, or usefulness of any information, apparatus, produce, or process disclosed, or represents that its use would not infringe privately owned rights. Reference herein to any specific commercial product, process, or service by trade aame, trademart, inanufacturer, or otherwise does not necessarily constizute or imply its endorsement, recommendation. or favoring by the United States Govesameat or any ageacy thereof. The views and opinions of authors expressed herein do not necessarily state or reflect those of the United States Governmeat or any agency thereof. 


\section{DISCLAIMER}

Portions of this document may be illegible in electronic image products. Images are produced from the best available original document. 


\section{NEUTRON DETECTOR RESOLUTION FOR SCATTERING \\ By}

Scott A. Kolda

A thesis Submitted to the Graduate

Faculty of Rensselaer Polytechnic Institute

in Partial Fulfillment of the

Requirements for the Degree of

MASTER OF SCIENCE

Approved:

$\frac{\text { Difeat Blah }}{\text { Dr.R.C. Block, Thesis Advisor }}$

Rensselaer Polytechnic Institute

Troy, New York

December, 1996 


\section{TABLE OF CONTENTS}

\section{Page}

LIST OF FIGURES

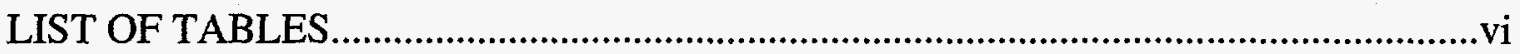

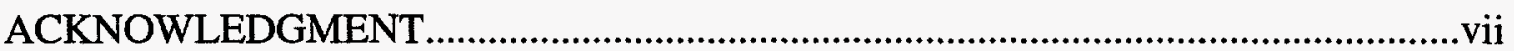

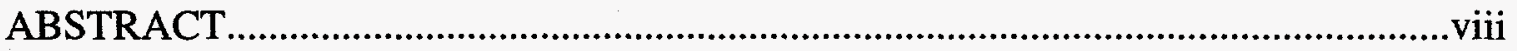

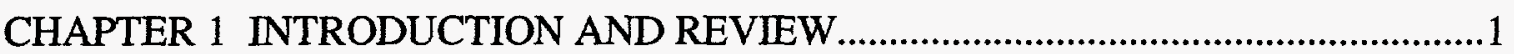

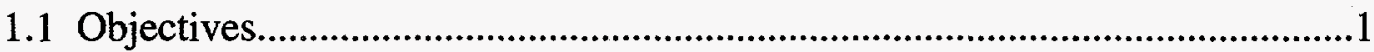

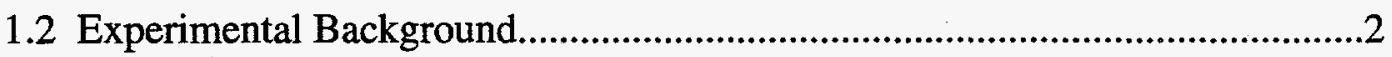

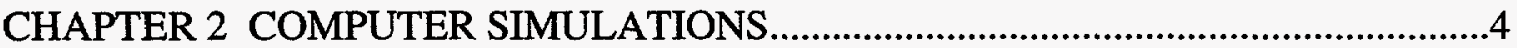

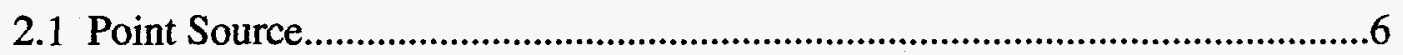

2.1.1 Point Source Analytical Solution..........................................................

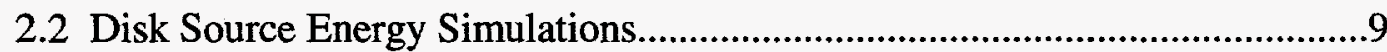

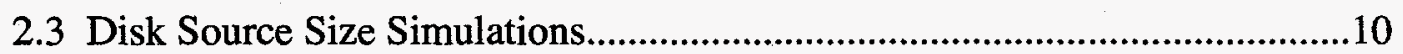

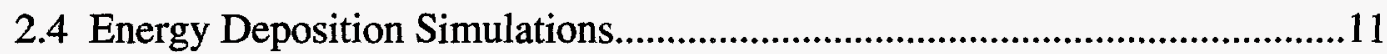

CHAPTER 3 RESOLUTION FUNCTION PARAMETERIZATION...............................14

3.1 Energy Dependence.....................................................................................19

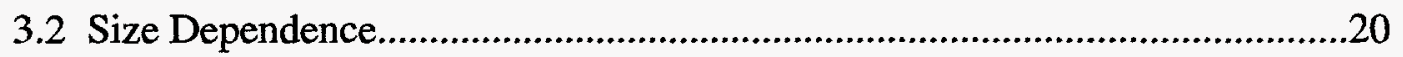

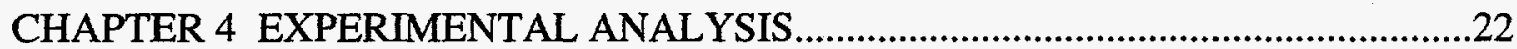

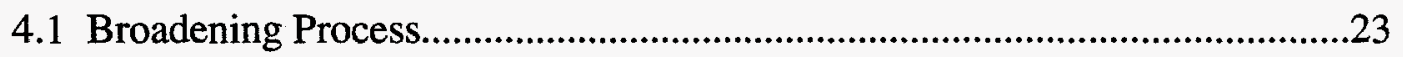

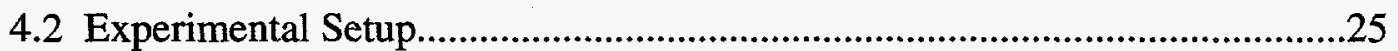

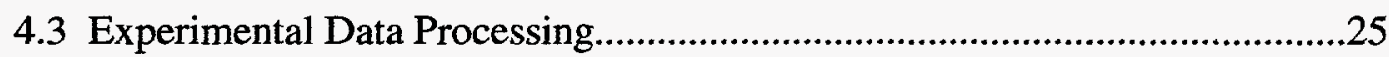




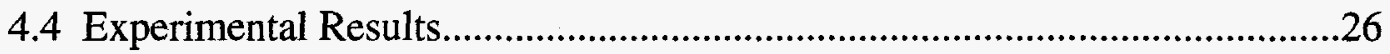

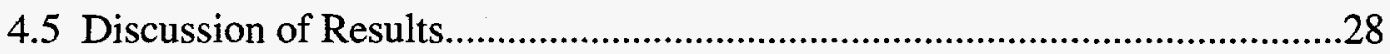

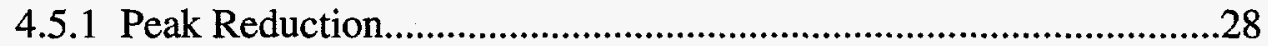

4.5.2 Lower Energy Wing Enhancement..........................................29

4.5.3 Scattering Target Resolution Function.....................................33

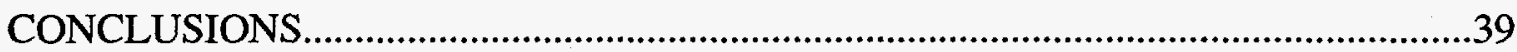

REFERENCES

APPENDIX A SAMPLE MCNP MODELING INPUT FLLES.................................43

A.1 Sample MCNP Input File for Disk Neutron Source Current on Inner Liner Surface

A.2 Sample MCNP Input File for Energy Deposition Determination..................44

APPENDIX B ${ }^{238}$ U RESONANCE FITS FOR DETECTOR RESOLUTION...............49

APPENDIX $C{ }^{238} \mathrm{U}$ RESONANCE FITS FOR COMBINED RESOLUTION................53 


\section{LIST OF FIGURES}

Figure

Figure 1.1 - A sketch of the 25 meter path for combined capture and scatter

experiments

Figure 1.2 - Multiplicity detector used for capture and scatter experiments. .3

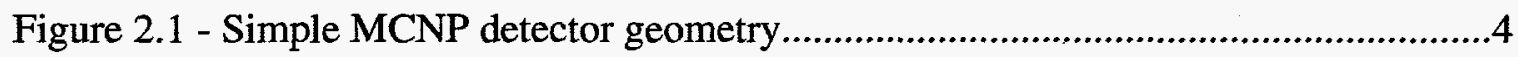

Figure 2.2 - Realistic MCNP detector geometry .......................................................5

Figure 2.3 - Point source neutron current incident on the inner liner surface for $10 \mathrm{eV}$

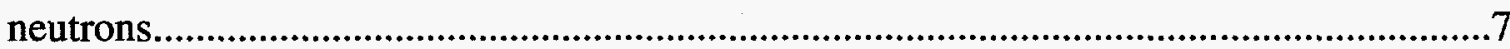

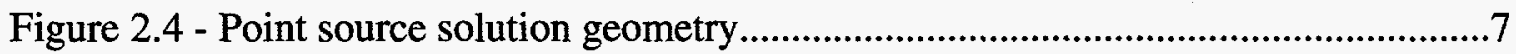

Figure 2.5 - Neutron current distribution in path length space for $1 \mathrm{eV}$ neutrons

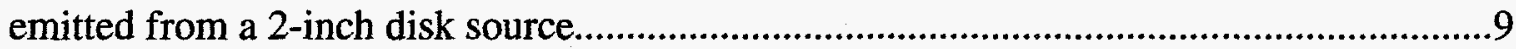

Figure 2.6 - Neutron current distribution in path length space for $1000 \mathrm{eV}$ neutrons

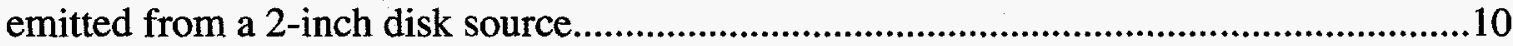

Figure 2.7 - Neutron intensity on the inner liner cylinder over time of $10 \mathrm{eV}$

neutrons for different sample disk sizes...........................................................11

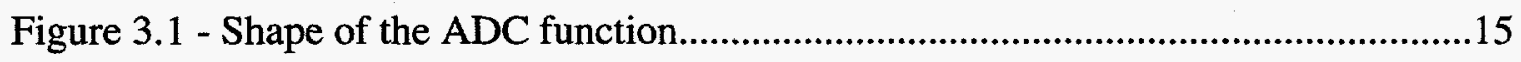

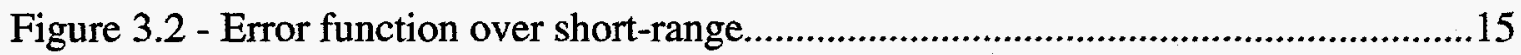

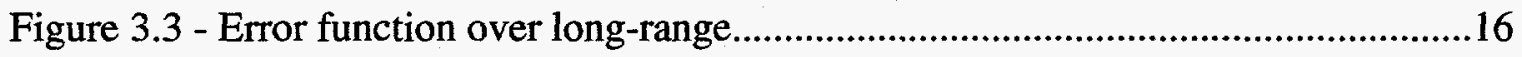

Figure 3.4 - Neutron count rate shift bulge curve fit for a 2-inch diameter disk $10 \mathrm{eV}$

neutron source. 
Figure 3.5 - Best fit for sum of exponentials curve for the longer time tail region for a 2-inch diameter disk $10 \mathrm{eV}$ neutron source.

Figure 3.6 - Combined curve fit of MCNP curve for neutron current incident upon inner detector liner surface for a 2 -inch diameter disk $10 \mathrm{eV}$ neutron source.

Figure 3.7 - Combined curve fit of MCNP curve for neutron current incident upon the inner detector liner surface for a 1 -inch diameter disk $10 \mathrm{eV}$ neutron source .20

Figure 4.1 - Calculated scattering yield from Doppler-broadened ENDF cross

sections for the $1 \mathrm{mil}^{238} \mathrm{U}$ sample

Figure 4.2 - Comparison of the resolution broadened ENDF calculated scattering yield and experimental scattering yield for the $102 \mathrm{eV}$ resonance for the $1 \mathrm{mil}$ ${ }^{238} \mathrm{U}$ sample

Figure 4.3 - Scatter detection efficiency sensitivity for the $102 \mathrm{eV}$ resonance for the $1 \mathrm{mil}{ }^{238} \mathrm{U}$ sample.

Figure 4.4 - Collision in the center-of-mass system where M1 is the incident particle

Figure 4.5 - Capture target resolution function at $189.7 \mathrm{eV}$ 35

Figure 4.6 - Experimental yield versus broadened ENDF calculated yield for the

${ }^{238} \mathrm{U} 189.7 \mathrm{eV}$ resonance. .36

Figure 4.7 - Scattering target resolution function for $189.7 \mathrm{eV}$ .37 


\section{LIST OF TABLES}

Table Page

Table 2.1 - MCNP determined photon energy deposition efficiency .12

Table 2.2 - Neutron leakage through the $\mathrm{B}_{4} \mathrm{C}$ liner.

Table 3.1 - Energy parameterized values.

Table 3.2 - Size parameterized values .21

Table 4.1 - Sample and number of triggers in capture/scatter experiment......................25

Table 4.2 - Resonance energy detector efficiency and fit values.................................27

Table 4.3 - Fraction of beam interacting at ${ }^{238} \mathrm{U}$ resonances for 1 mil sample.................29

Table 4.4 - Calculated measured energy shift due to nuclear recoil for ${ }^{238} \mathrm{U}$.....................31

Table 4.5 - Calculated measured energy shift due to nuclear recoil for Mo.....................32

Table 4.6 - Calculated measured energy shift due to nuclear recoil for Fe.......................32

Table 4.7 - Constants used in capture and scattering target resolution functions..............35

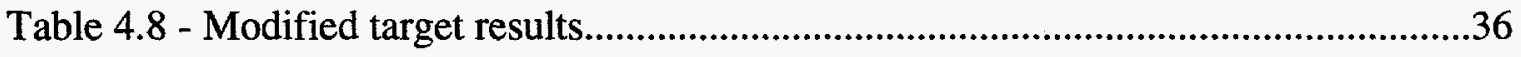




\section{ACKNOWLEDGMENTS}

I am grateful to Dr. R. C. Block, my thesis advisor, who introduced me to the topic and provided the guidance necessary to complete the thesis. I am also grateful to Dr. R. E. Slovacek, who was always available for help and kept me pointed in the right direction.

Many thanks to Dr. N. Francis, without whose help I would still be working on the initial problem. Thank you to Dr. J. Burke for the installation of the MCNP files. Thank you to N. Drindak for the necessary detector information. Thank you to Dr. G. Leinweber for the previously written detector program and additional insight into the modeling program. Thank you to D. Mesh for your support.

I would like to also thank the LINAC staff of J. Westhead, P. Brand, M. Gray and L. Prince for their support.

Thank you to my fellow students including B. Moretti, C. Werner and M. Overberg. I would like to especially thank B. Moretti and C. Werner who were always helpful in answering my myriad of questions ranging from MCNP to data analysis.

Finally, I would like to thank my family for putting up with the past year. Without the support of Jessica, Zachary and Jacob, this work would have not been possible. 


\begin{abstract}
A resolution function has been determined for scattered neutron experiments at Rensselaer Polytechnic Institute (RPI). This function accounts for the shifting and broadening of the resonance peak due to the additional path length, traveled by the neutron after scattering and prior to detection, along with the broadening of the resonance peak due to the bounce target. This resolution function has been parameterized both in neutron energy and size of the sample disk.
\end{abstract}

Monte Carlo Neutron and Photon (MCNP) modeling has been used to determine the shape of the detector resolution function while assuming that the sample nucleus has an infinite mass. The shape of the function for a monoenergetic neutron point source has been compared to the analytical solution. Additionally, the parameterized detector resolution function has been used to broaden the scatter yield calculated from Evaluated Neutron Data File ENDF/B-VI cross section data for ${ }^{238} \mathrm{U}$.

The target resolution function has been empirically determined by comparison of the broadened scatter yield and the experimental yield for ${ }^{238} \mathrm{U}$.

The combined resolution function can be inserted into the SAMMY code to allow resonance analysis for scattering measurements. 


\section{CHAPTER 1}

\section{INTRODUCTION AND REVIEW}

\subsection{Objectives}

The Rensselaer Polytechnic Institute (RPI) linear accelerator (LINAC) facility uses the neutron time-of-flight method in measuring the transmission, capture and scattering of neutrons. Applying the Oak Ridge National Laboratory neutron data analysis code $\mathrm{SAMMY}^{1}$, only the resonance parameters from transmission and capture measurements are able to be determined today. The scattered neutrons travel an additional path length after interacting with the nuclei of the material sample which results in a distortion of the resonance shape. This distortion of the resonance shape has been previously undetermined.

The function describing the resonance shape distortion is to be incorporated into the SAMMY code. This function and the scattered neutron detector efficiency are both determined using the standard RPI 2-inch diameter disk sample for a neutron source energy range of 1 to $1000 \mathrm{eV}$. The resolution function dependence on sample size has been determined for sample sizes ranging from 0.25 to 2 inches in diameter for a neutron source energy of $10 \mathrm{eV}$ since samples may be smaller than the standard size due to reasons of availability or expense. 


\subsection{Experimental Background}

The RPI LINAC produces pulses of high energy electrons. These electrons then strike a tantalum target producing a neutron source. The electrons decelerate in the target through Coulombic interactions resulting in the emission of high energy bremsstrahlung photons. These photons undergo photoneutron interactions with the tantalum, generating free neutrons which are emitted nearly isotropically from the target. A portion of these neutrons are moderated and then pass through the collimated flight tubes to where they interact with the sample material of interest. The transmission, capture and scattering of these neutrons are measured and the resonance parameters are later determined. The configuration for capture and scattering measurements is shown in figure 1.1.

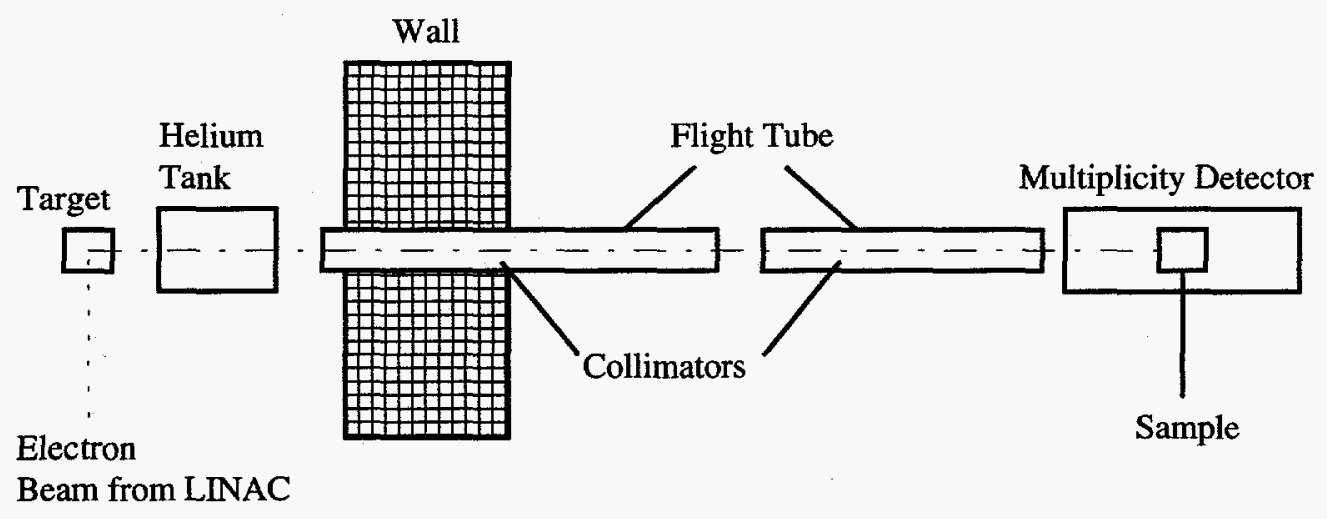

Figure 1.1 - A sketch of the 25 meter path for combined capture and scatter experiments

The detector used at RPI for combined neutron capture and scatter experiments is a sixteen section $\mathrm{NaI}$ detector which is shown in figure $1.2^{2}$. 


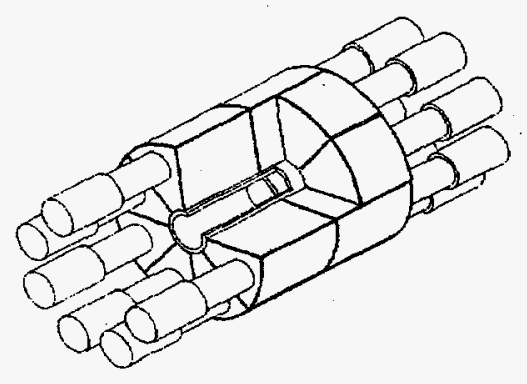

Figure 1.2 - Multiplicity detector used for capture and scatter experiments

Neutrons scattered from the sample are absorbed by boron present in the $99.46 \%$ ${ }^{10} \mathrm{~B}$ enriched $\mathrm{B}_{4} \mathrm{C}$ liner in the following reactions:

$$
\begin{aligned}
& { }^{10} B+n \rightarrow{ }^{7} L i^{*}+\alpha ;{ }^{7} L i^{*} \rightarrow{ }^{7} L i+\gamma \\
& { }^{10} B+n \rightarrow{ }^{7} L i+\alpha
\end{aligned}
$$

The resulting gamma, which is created $93 \%$ of the time, has an energy of $477 \mathrm{keV}$. This gamma can enter the NaI detector crystals, where it is absorbed. If the energy absorbed in the crystal is between the detector discriminator setpoints of 360 and $600 \mathrm{keV}$, it is then recorded as a scattered neutron. These discriminator setpoints were chosen to limit background in the recorded scattering data.

The Monte Carlo Neutron and Photon (MCNP) code simulation solution is tested through two methods. In the first method, a point source is modeled in MCNP. The time distribution of neutrons on the inner surface of the liner is then compared to the analytical solution. In the second method, the resolution function for a 2-inch diameter isotropic source (infinite mass approximation) is tested for accuracy using experimental scattering data from the ${ }^{238} \mathrm{U} 1 \mathrm{mil}(0.001$ ") sample recorded October 11 to $12,1995$. 


\section{CHAPTER 2}

\section{COMPUTER SIMULATIONS}

The primary means of determining the scattered neutron path length distribution has been through the Monte Carlo Neutron and Photon (MCNP) 4A transport code ${ }^{3}$ developed at the Los Alamos National Laboratory and distributed through the Radiation and Shielding Information Center (RSIC) at the Oak Ridge National Laboratory. The MCNP code simulates the movement of individual particles and records certain aspects of their behavior. This code is used in determining the time distribution of neutrons on the inner surface of the detector liner, the time distribution of gamma rays exiting the outside radius of the liner, and the scattered neutron detection efficiency.

MCNP allows for both simple and complicated geometries used in detector modeling. The cross sections included in the code allow for both neutron-photon simulations and energy deposition determination. The simple geometry used for the detector liner is shown below in figure 2.1 and the more realistic complicated geometry used for the detector is shown in figure 2.2 .

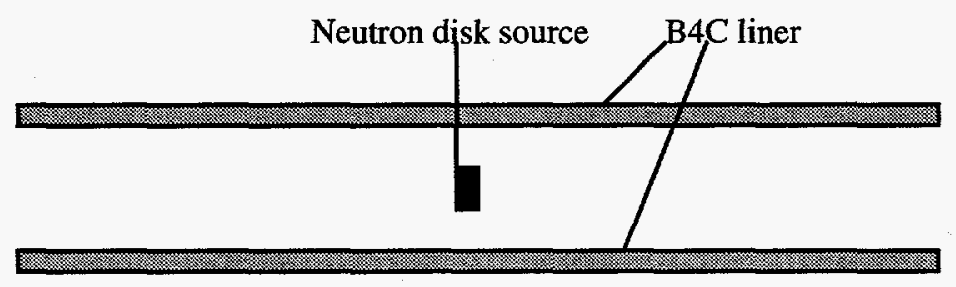

Figure 2.1 - Simple MCNP detector geometry 

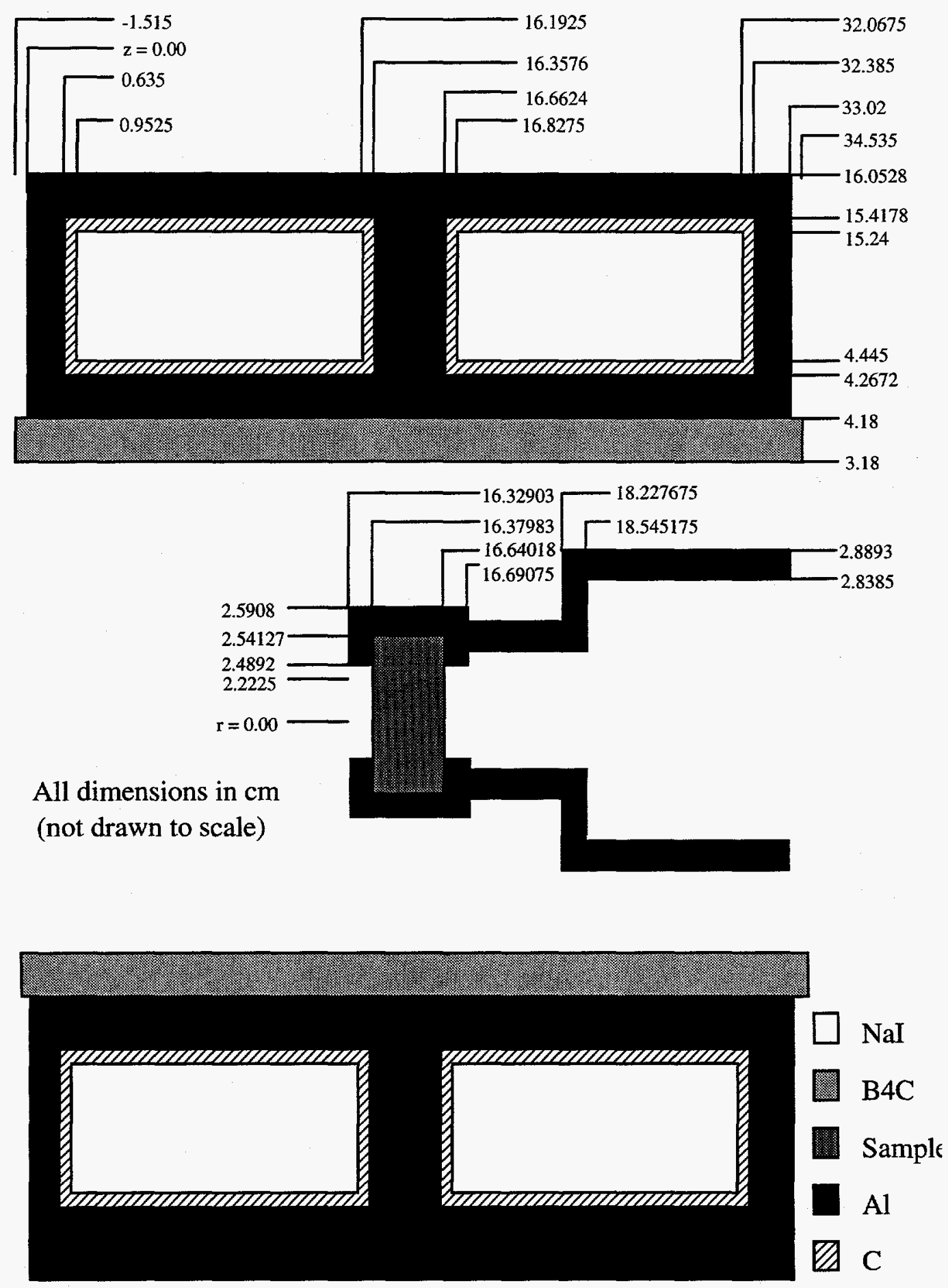

Figure 2.2 - Realistic MCNP detector geometry 
The detector geometry is an updated version of the model used by Leinweber ${ }^{4}$. The liner has been upgraded to ${ }^{10} \mathrm{~B}_{4} \mathrm{C}$ ceramic that extends beyond the detector by 1.515 $\mathrm{cm}$ on each end. Additionally, the NaI detector crystals had been previously modeled as NaSn due to the lack of an I cross section file. The current detector model has ${ }^{127} \mathrm{I}$ included.

\subsection{Point Source}

The initial simple detector model consists of a monoenergetic $10 \mathrm{eV}$ neutron point source located at the center of the detector and the detector liner. A total of 100,000 case histories have been run with the neutron current entering the inner surface of the liner being scored. The results of this simulation are shown in figure 2.3 with the analytical solution fitted to the MCNP data, where 1 shake is $10^{-8}$ second. The time distribution of neutrons on the inner surface of the detector liner has an asymptotic nature which is explained by the analytical model. 


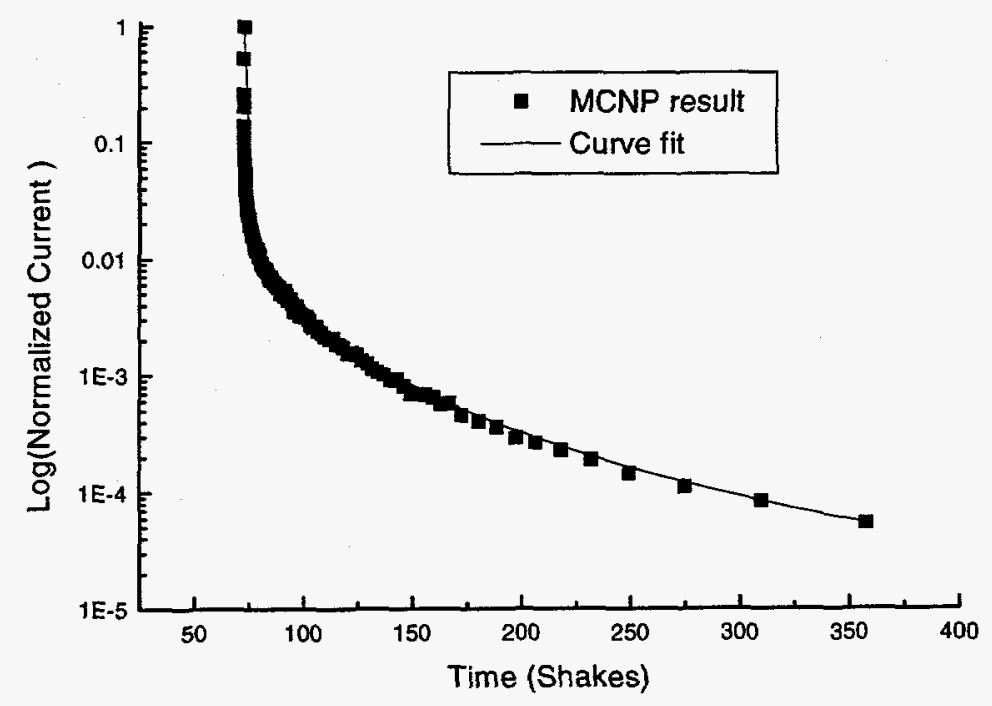

Figure 2.3 - Point source neutron current incident on the inner liner surface for $10 \mathrm{eV}$ neutrons

\subsubsection{Point Source Analytical Solution}

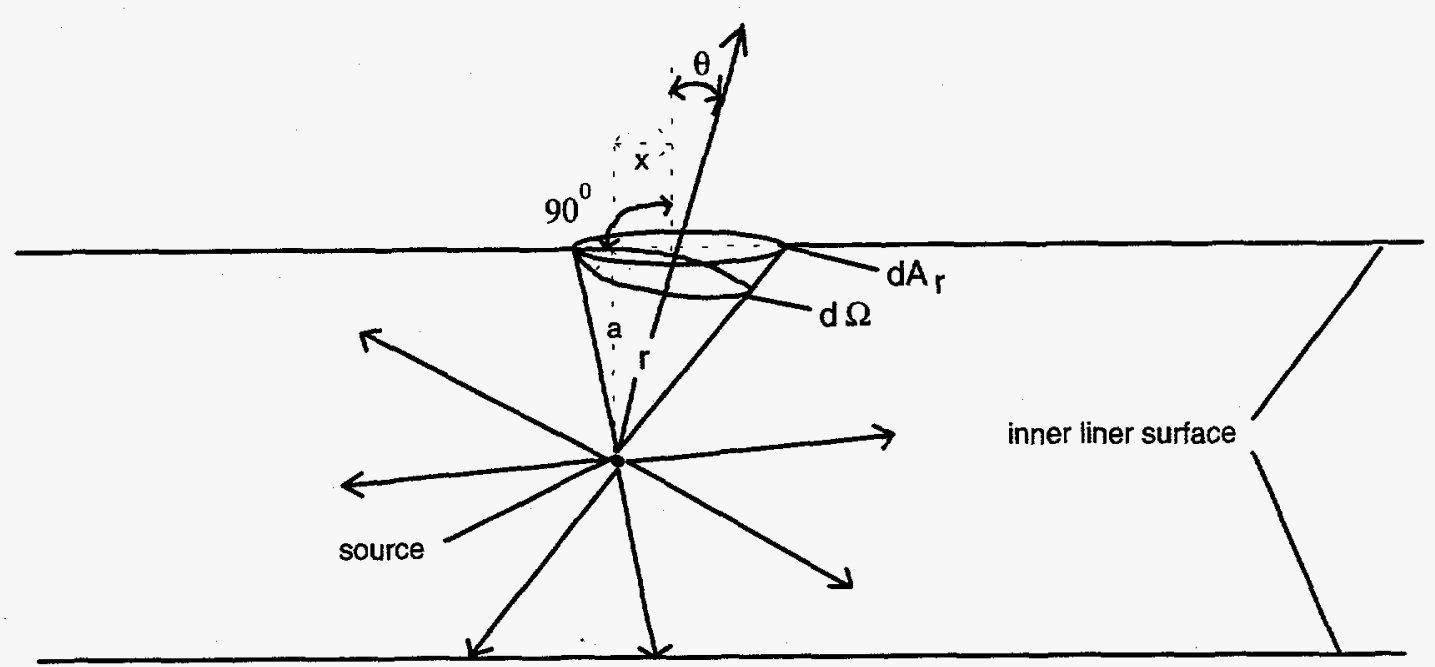

Figure 2.4 - Point source solution geometry 
The proof follows the method used by Maissel and Glang ${ }^{5}$ in describing metal deposition on a planar surface after evaporation from a point source. The geometry used for the analytical solution is shown in figure 2.4. The value of $I_{r}$ is the value of neutron current intensity across the inner surface of the detector liner, with inner radius a, as a function of path length.

$$
d A_{r}=\frac{d \Omega \cdot r^{2}}{\cos \theta}
$$

The distance from the point source to the inner liner surface is $r$ and the remaining symbols are shown in figure 2.4 .

$$
\begin{gathered}
d I_{r}=\frac{d \Omega}{4 \pi}=\frac{d A_{r} \cdot \cos \theta}{4 \pi \cdot r^{2}} \\
d A_{r}=2 \pi a \cdot d x \\
x=\sqrt{r^{2}-a^{2}} \\
d x=\frac{r \cdot d r}{\sqrt{r^{2}-a^{2}}} \\
\cos \theta=\frac{a}{r} \\
d I_{r}=\frac{a^{2} \cdot d r}{2 r^{2} \sqrt{r^{2}-a^{2}}}
\end{gathered}
$$

The conversion of equation (2.7) from path length space to time space is fairly simple since the neutrons are monoenergetic. By substituting $r=v t$ and $a=v t_{0}$ into equation (2.7), where $v$ is the velocity, the time distribution of intensity becomes:

$$
d I_{t}=\frac{t_{0}^{2} d t}{2 t^{2} \sqrt{t^{2}-t_{0}^{2}}}
$$


The curve in figure 2.3 is the fit to the MCNP data using equation (2.8)

\subsection{Disk Source Energy Simulations}

The next series of simple geometric simulations were carried out for a 2 inch diameter disk sample as shown in figure 2.2 and with a parallel beam of incoming neutrons from the left. These neutrons scatter isotropically from the sample nuclei which are assumed to have infinite mass. Monoenergetic beam neutrons of $1,10,100$, and 1000 $\mathrm{eV}$ were used in four separate simulations. For these simulations, 1 million neutron case histories were followed into the $\mathrm{B}_{4} \mathrm{C}$ liner and the photon current exiting the outer liner radius was scored. The sample input for the $10 \mathrm{eV}$ case is presented in Appendix A.1. In figures 2.5 and 2.6, the time distribution of the photon current is shown for normalized 1 and $1000 \mathrm{eV}$ source neutrons; these are displayed as a function of the product of time and square root of energy. There is an apparent shift toward longer path length for the higher energy neutrons. This will be shown to be a minor effect in Chapter 3 .

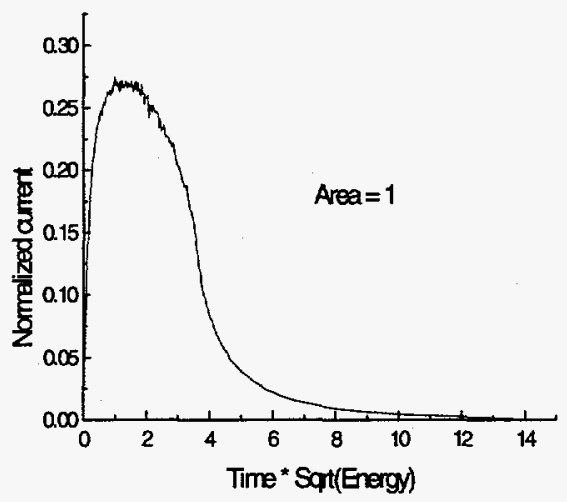

Figure 2.5 - Neutron current distribution in path length space for $1 \mathrm{eV}$ neutrons emitted from a 2-inch disk source. 


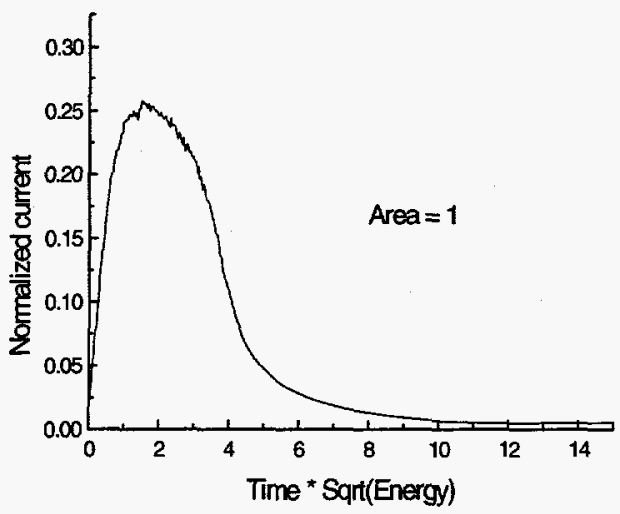

Figure 2.6 - Neutron current distribution in path length space for $1000 \mathrm{eV}$ neutrons emitted from a 2-inch disk source.

\subsection{Disk Source Size Simulations}

The following series of simulations concern the relationship between scattered neutron path length and sample size. Simulations were carried out for monoenergetic 10 $\mathrm{eV}$ neutron isotropic sources of $0.25,0.5,1,1.5$ and 2 inches in diameter. The point source is excluded due to the singularity in intensity. For these simulations, 1 million neutron case histories were followed to the inner surface of the liner, where the neutron current was scored. Figure 2.7 demonstrates the change in neutron current intensity over time as a function of disk diameter. The significant effect of disk size on the distribution of path lengths is apparent. Disk sizes less than 1 inch in diameter show a relatively narrow peak when compared to the standard 2-inch disk size. This makes it difficult to find a universal function to describe these curves. That process will be covered in Chapter 3. 


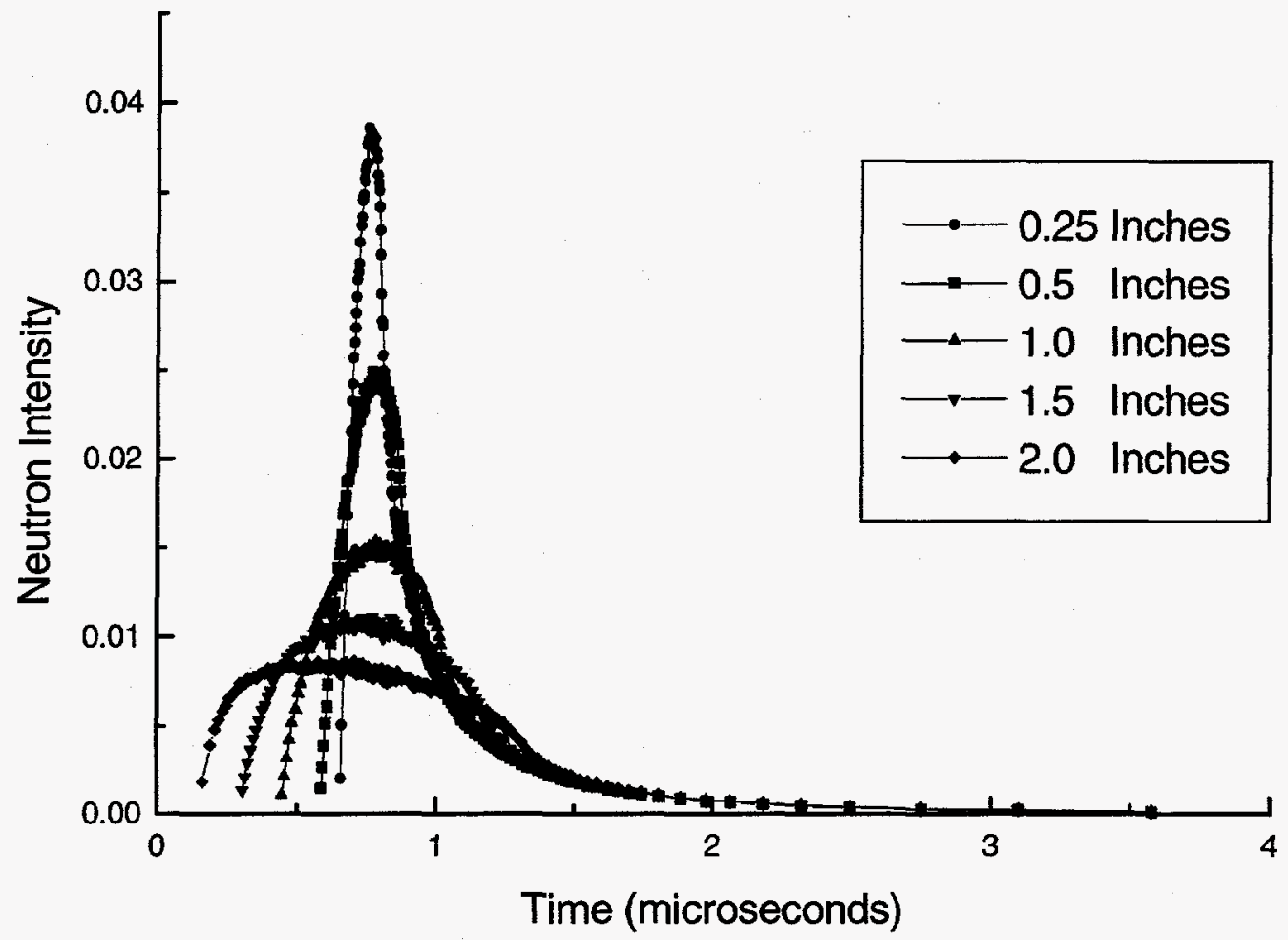

Figure 2.7 - Neutron intensity on the inner liner cylinder over time of $10 \mathrm{eV}$ neutrons for different sample disk sizes

\subsection{Energy Deposition Simulations}

Not all photons exiting the outer $\mathrm{B}_{4} \mathrm{C}$ liner surface are going to be detected. First of all, the liner extends beyond the detector crystals. The number of neutrons captured in this region is small. Second, the photons could travel through the crystal without interaction. This is unlikely for the NaI crystal. Third, not all energy depositions in the crystal are recorded as scattering events. Discriminator setpoints of $360 \mathrm{keV}$ and $600 \mathrm{keV}$ have been established to prevent corruption of the scattering signal. The higher limit denotes the cutoff between the low energy scattering signal photons, which have a birth 
energy of $477 \mathrm{keV}$, and the high energy capture signal photons, which are born at energies greater than $1 \mathrm{MeV}$. The lower setpoint was established to minimize the effects of system background. Approximately $28 \%$ of the scatter signal photons are Compton scattered in the liner to energies less than $360 \mathrm{keV}$.

The energy deposition simulations were carried out for monoenergetic scattered neutron energies of $1,10,100$ and $1000 \mathrm{eV}$ neutrons. For each simulation, the scattered neutron was followed into the liner, where the radiative absorption took place. The photons were then transported into the crystals, where the energy deposition was scored. One million neutron case histories were run at each of the neutron energies. The simulation input for the $10 \mathrm{eV}$ neutron source is included in Appendix A.2. The simulation results are shown in Table 2.1 .

\begin{tabular}{|c|c|c|}
\hline Neutron source energy (eV) & $\begin{array}{c}\text { Photon energy deposition range } \\
(\mathrm{keV})\end{array}$ & Fraction of events \\
\hline 1 & & \\
\hline & $<360$ & $0.229 \pm 0.0015$ \\
\hline & $360-680$ & $0.587 \pm 0.0008$ \\
\hline 10 & & $0.295 \pm 0.0015$ \\
\hline & $<360$ & $0.575 \pm 0.0009$ \\
\hline 100 & $360-680$ & $0.288 \pm 0.0016$ \\
\hline & & $0.550 \pm 0.0009$ \\
\hline & $<360$ & \\
\hline 1000 & $360-680$ & $0.260 \pm 0.0017$ \\
\hline & & $0.489 \pm 0.0010$ \\
\hline
\end{tabular}

Table 2.1 - MCNP determined photon energy deposition efficiency 
As can be seen in Table 2.1, approximately $16 \%$ of the neutrons below $100 \mathrm{eV}$ emitted from the source do not deposit any energy into the detector crystals. MCNP simulations measuring different tallies have been performed to determine the nature of the losses. Since the detector liner is $36.05 \mathrm{~cm}$ long, MCNP simulations reveal that $1.8 \%$ of the neutrons escape out the ends of the detector liner without being captured. As mentioned earlier, $7 \%$ of the neutron capture reactions with ${ }^{10} \mathrm{~B}$ do not produce photons and will not be counted. Additionally, $1-2 \%$ of the photons that have been produced by the ${ }^{10} \mathrm{~B}$ captures in the liner escape out the ends of the detector liner. The remaining signal loss of approximately $4 \%$ is internal to the detector.

The additional signal loss at higher neutron energies is due to neutron leakage through the detector liner. This neutron leakage has been determined for the 2 -inch disk source by using the simple model. The neutron current exiting the outer liner surface has been scored for one million case histories. The detector liner captures a very high percentage of the incident neutrons over the energy range of interest, 1 to $200 \mathrm{eV}$. This can be seen in Table 2.2 .

\begin{tabular}{|c|c|}
\hline Neutron source energy (eV) & $\begin{array}{c}\text { Fraction of neutrons exiting } \\
\text { liner }\end{array}$ \\
\hline 1 & 0 \\
\hline 10 & 0 \\
\hline 100 & $0.000327 \pm 0.0542$ \\
\hline 1000 & $0.0555 \pm 0.004$ \\
\hline
\end{tabular}

Table 2.2 - Neutron leakage through the $\mathrm{B}_{4} \mathrm{C}$ liner 


\section{CHAPTER 3}

\section{RESOLUTION FUNCTION PARAMETERIZATION}

Successful incorporation of a scattering resolution function into the SAMMY code for the standard RPI sample size of a 2-inch diameter disk requires energydependent parameterized curves. To allow for different size samples to be used, sizeparameterized curves for the scattering resolution function will also be determined.

The curve fitting program of TableCurve $2 \mathrm{D}^{6}$ was used to assist in this task. This program uses automated statistical methods to determine the best curve fit from a library of 3456 linear and nonlinear equations. The equation which showed the most promise for fitting the MCNP resultant time distribution of neutrons or photons over all disk sizes and energies is the asymmetric double cumulative (ADC) equation. The curve, the product of two error functions, has the equation and shape shown below, where a, b, c, d, e and $f$ are the constants determined during fitting.

$$
y=a+\frac{b}{4}\left[1+e r f\left(\frac{x-c+d / 2}{e \sqrt{2}}\right)\right]\left[1-e r f\left(\frac{x-c-d / 2}{f \sqrt{2}}\right)\right]
$$




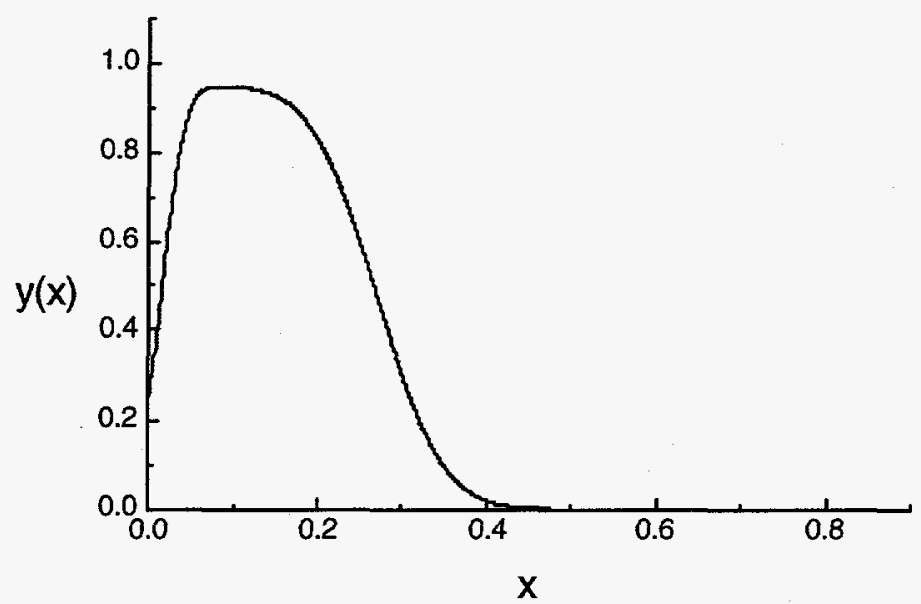

Figure 3.1 - Shape of the ADC function

The error function, $\operatorname{erf}(\mathrm{x})$, is described by equations 3.2 and 3.3 below $^{7}$. Figures 3.2 and 3.3 demonstrate the behavior of the error function for two different ranges of the independent parameter. As can be seen below, the value of erf(x) approaches \pm 1 as

$$
\begin{aligned}
& \operatorname{erf}(x)=\frac{2}{\sqrt{\pi}} \int_{0}^{x} e^{-t^{2}} d t \\
& \operatorname{erf}(-x)=-\operatorname{erf}(x)
\end{aligned}
$$

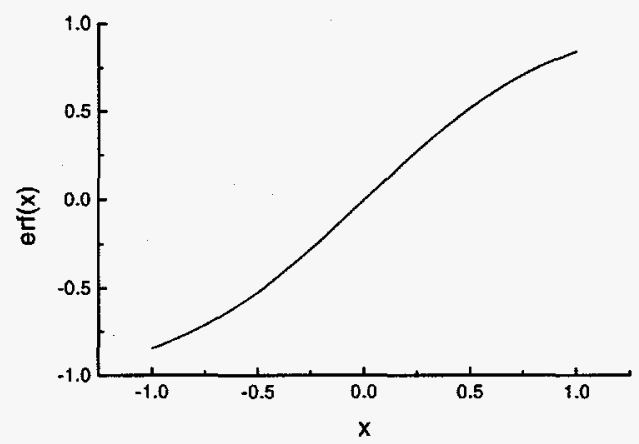

Figure 3.2 - Error function over short-range 


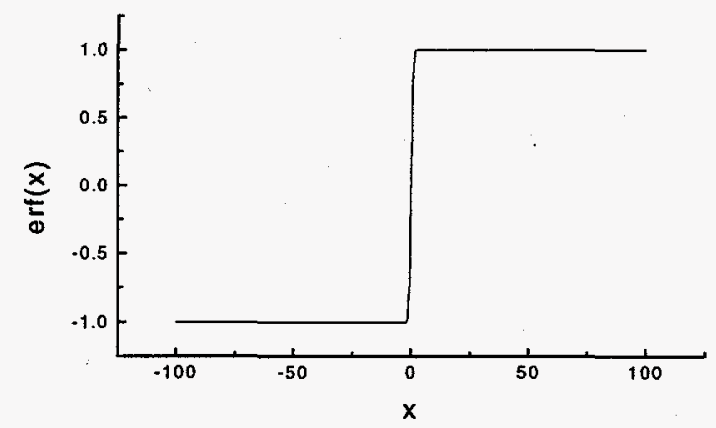

Figure 3.3 - Error function over long-range

the value of $x$ approaches infinity in either direction. Therefore, the value of the ADC curve approaches the value of ' $a$ ' in this situation. The ADC function has been modified in the following manner to use as a resolution function. The value of ' $a$ ' has been set to zero to have the curve cover a finite area as $\mathrm{x}$ approaches infinity. The resulting function has been termed the "bulge" equation and has the form of equation (3.4) below.

$$
y=b\left[1+e r f\left(\frac{x-c+d / 2}{e \sqrt{2}}\right)\right]\left[1-e r f\left(\frac{x-c-d / 2}{f \sqrt{2}}\right)\right]
$$

The bulge equation has been fitted to a selected portion of the MCNP curves by using the Origin ${ }^{8}$ curve fitting program. This method allows the bulge curve to reproduce as much of the MCNP curve as possible. The comparison of the two curves is shown in figure 3.4. 


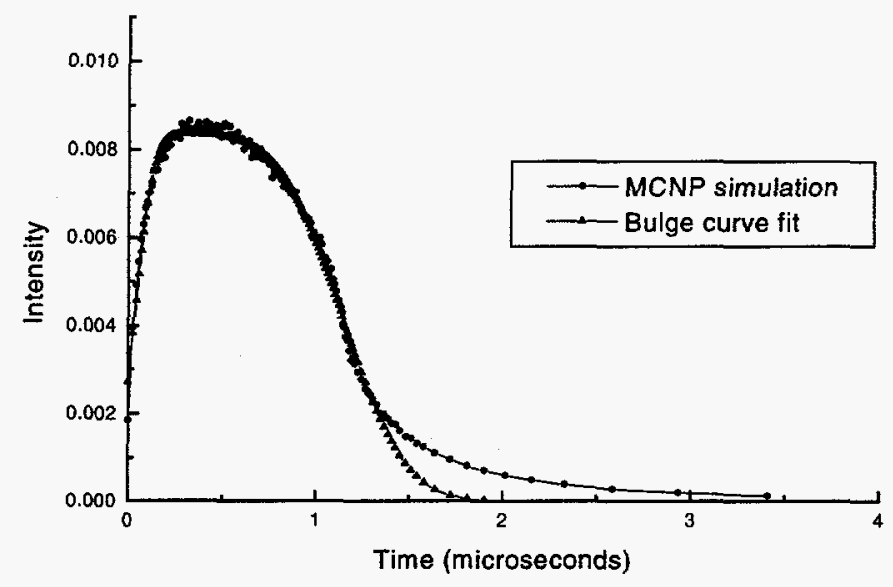

Figure 3.4 - Neutron count rate time shift bulge curve fit for a 2-inch diameter disk $10 \mathrm{eV}$ neutron source

As can be seen above, the bulge curve drops off faster than the MCNP simulation resultant shape. Figure 3.5 below shows the shape of the difference between the two curves for the greater time region. This shape has been fitted in Origin using a sum of two exponentials in the form of equation (3.5). This equation, previously used by Moretti ${ }^{9}$ to modify the neutron target resolution equation in SAMMY, has been termed the "tail" . curve and is shown in equation (3.5) below, where $\alpha, \beta, \gamma, \delta, \varepsilon$ and $\tau$ are the constants determined during fitting.

$$
\operatorname{tail}(t)=\alpha\{\cdot \beta \exp [\gamma(t+\tau)]+\delta \cdot \exp [\varepsilon(t+\tau)]\}
$$




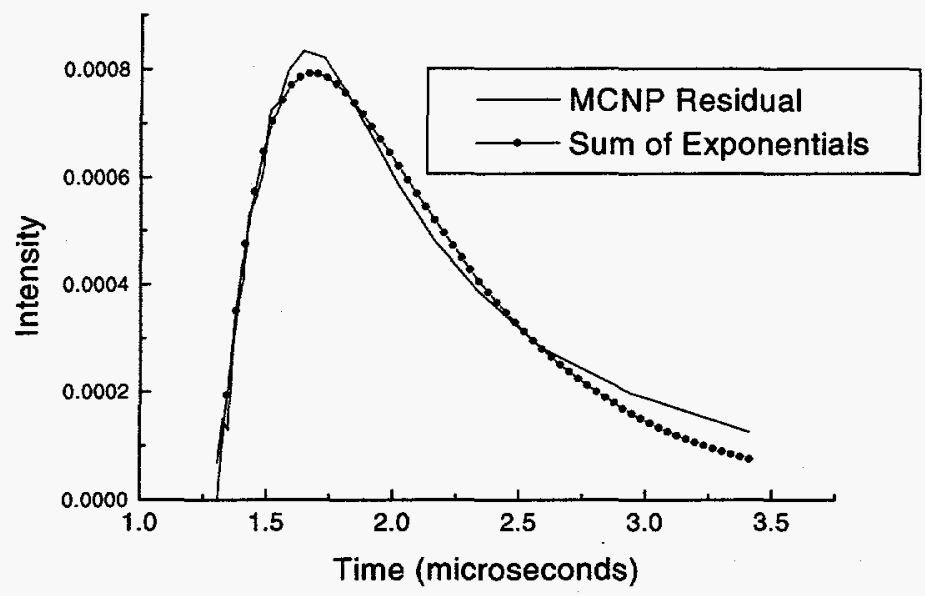

Figure 3.5 - Best fit of sum of exponentials curve for the longer time tail region for a 2 -inch diameter disk $10 \mathrm{eV}$ neutron source

Following the method of Moretti, the two equations are summed together starting at the point where the value of the tail equation is zero. A plot of the MCNP and combined tail and bulge curves is shown in figure 3.6. As can be seen, the MCNP curve has been well represented as a sum of the two equations.

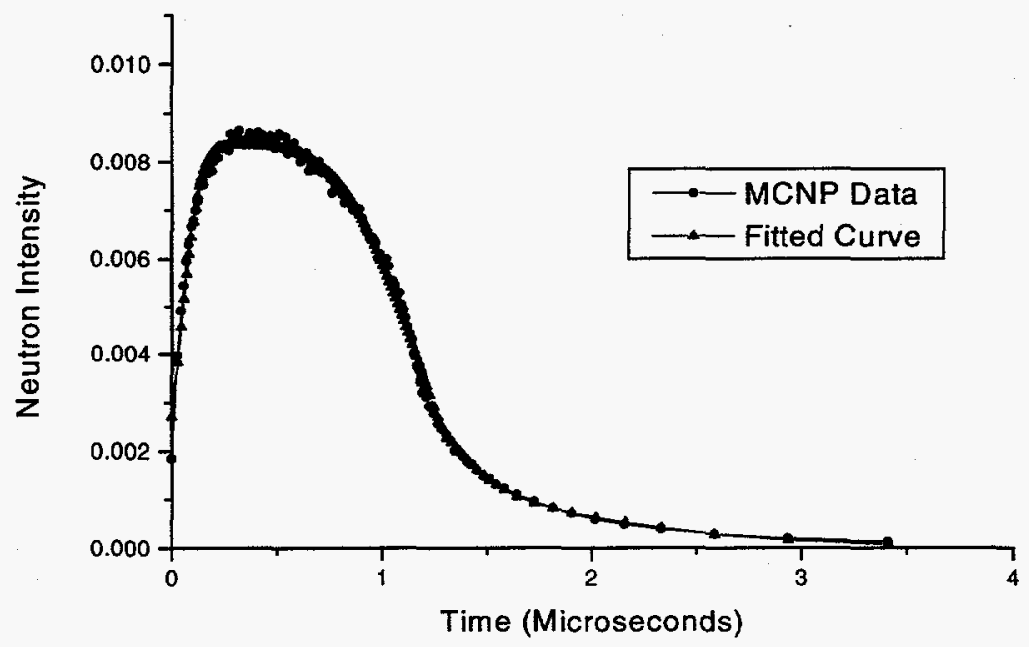

Figure 3.6 - Combined curve fit of MCNP curve for neutron current incident upon inner detector liner surface for a 2-inch diameter disk $10 \mathrm{eV}$ neutron source 


\subsection{Energy Dependence}

The constants in both the bulge and tail curves were parameterized as a function of energy over an energy range of 1 to $1000 \mathrm{eV}$ for a 2 -inch neutron disk source for photon current exiting the outer liner surface. The points used in the parameterization were the $1,10,100$ and $1000 \mathrm{eV}$ values for the parameters in equations (3.4) and (3.5). The result is shown in table 3.1. The parameterized curves were determined by Origin for the simpler curves and TableCurve 2D for the more sensitive parameters.

\begin{tabular}{|c|}
\hline BULGE CURVE \\
\hline$b=7.932\left(10^{-4}\right) \cdot E^{0.4563}-1.843\left(10^{-4}\right)$ \\
\hline$c=1.877 \cdot E^{-0.5}+0.0054$ \\
\hline$d=3.468 \cdot E^{-0.5}+0.00432$ \\
\hline$e=0.216 \cdot E^{-0.5}+0.00563$ \\
\hline$f=0.854 \cdot E^{-0.5}+4.827\left(10^{-4}\right)$ \\
\hline TAIL CURVE \\
\hline$\alpha=-0.2742 \cdot E^{-0.0486}+0.2751$ \\
\hline$\beta=1.6044\left(10^{-4}\right)-0.5876$ \\
\hline$\gamma=-1.221 \cdot E^{0.5}-0.1788$ \\
\hline 1 \\
\hline$\delta=\frac{1.9612-0.1557 \cdot \ln (E)-0.08541 \cdot E^{-2}}{2 \varepsilon=-0.5266 \cdot E^{0.5}+0.02379}$ \\
\hline$\tau=-4.057 \cdot E^{-0.5}-0.05542$ \\
\hline
\end{tabular}

Note: $\mathrm{E}$ is energy in $\mathrm{eV}$

Table 3.1 - Energy parameterized values

Parameter sensitivity was determined by visually comparing the MCNP fitted curves with the parameterized value curves in Mathcad ${ }^{10}$. The dependence of the values would be expected to be a function of energy in the manner of $\mathrm{E}^{0.5}$ or $\mathrm{E}^{-0.5}$ since the time required 
for the scattered neutrons to reach the inner surface of the liner is proportional to $\mathrm{E}^{-0.5}$. This is the case for most of the parameters. As a note, the areas of the curves have not been normalized to a value of 1 and need to be normalized prior to application. The applicability of these curves will be explored in chapter 4 where an interpolated curve will be tested against the ${ }^{238} \mathrm{U} 36 \mathrm{eV}, 66 \mathrm{eV}, 102 \mathrm{eV}$ and $189 \mathrm{eV}$ resonance scattering data.

\subsection{Size Dependence}

The constants in both the bulge and tail curves were parameterized over a diameter range from 0.25 to 2 inches for a $10 \mathrm{eV}$ neutron disk source for neutron current entering the inner liner surface. The fitted curve is shown in Figure 3.7 for the 1 -inch disk.

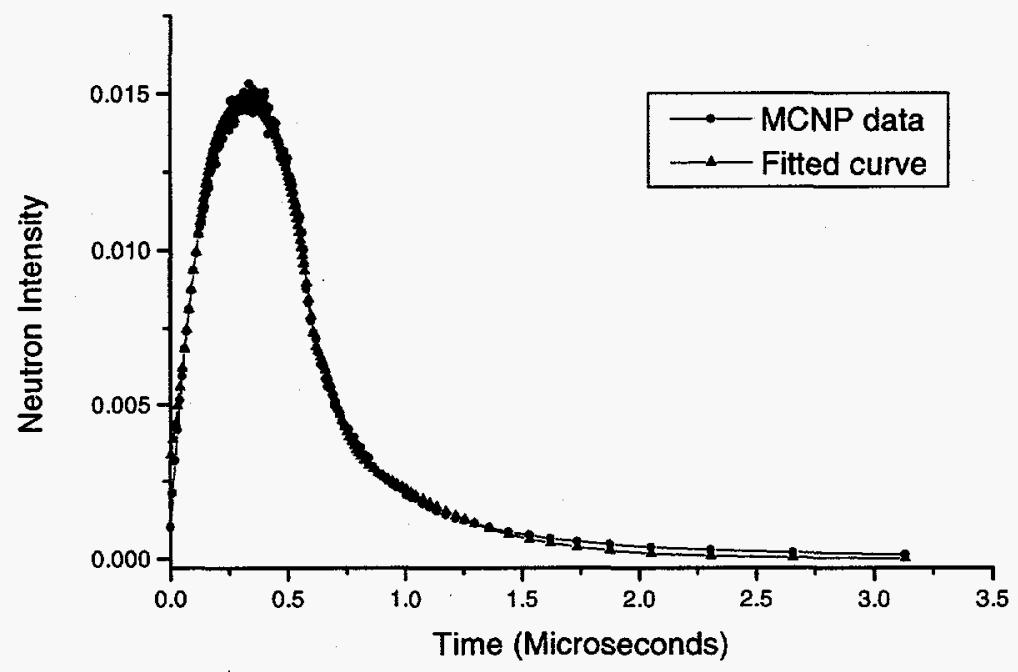

Figure 3.7 - Combined curve fit of MCNP curve for neutron current incident upon the inner detector liner surface for a 1 -inch diameter disk $10 \mathrm{eV}$ neutron source 
The points used in the parameterization were the $0.25,0.5,1.0,1.5$ and 2.0-inch disk values for the parameters in equations (3.4) and (3.5). The result is shown in table 3.2. The parameterized curves were determined by Origin for the simpler curves and TableCurve 2D for the more sensitive parameters. Parameter sensitivity was determined by visually comparing the MCNP fitted curves with the parameterized value curves in Mathcad.

\begin{tabular}{|c|}
\hline BULGE CURVE \\
\hline$b=\frac{1}{475.81 \cdot D^{0.5}-197.55}$ \\
\hline$c=\frac{1}{0.4425+\frac{2.4911}{D}}$ \\
\hline$d=\sqrt{0.3097 \cdot D^{2}-0.01796}$ \\
\hline$e=0.1131+0.05961 \cdot D \ln D-0.02113 \cdot D^{2.5}+0.001941 \cdot \frac{\ln D}{D^{2}}$ \\
\hline$f=0.03049 \cdot D^{2.634}+0.07878$ \\
\hline
\end{tabular}

\begin{tabular}{|c|}
\hline$\alpha=\sqrt{1.0101\left(10^{-5}\right)-2.1283\left(10^{-5}\right) \cdot D+1.3606\left(10^{-5}\right) \cdot D^{2}-9.6377\left(10^{-7}\right) \cdot D^{3}}$ \\
\hline$\beta=\frac{1}{0.1661-13.070 \cdot \exp (-D)}$ \\
\hline$\gamma=-8.2784+5.900 \cdot \ln D$ \\
\hline$\delta=-1.1780 \cdot D+2.9307$ \\
\hline$\varepsilon=2.3820 \cdot D^{0.5}-5.0290$ \\
\hline$\tau=-1.5676+1.4699 \cdot \exp (-D)$ \\
\hline
\end{tabular}

Note: $\mathrm{D}$ is disk diameter in inches

Table 3.2 - Size parameterized values 


\section{CHAPTER 4}

\section{EXPERIMENTAL ANALYSIS}

The resolution function was tested by comparing scattered neutron data measured for ${ }^{238} \mathrm{U}$ on October $11-12,1995$ with ENDF/B-VI (Evaluated Neutron Data File B, version VI) cross sections. Uranium was chosen since its resonance parameters are known to a fairly high level of confidence. Also, the heavy nucleus can be approximated as having an infinite mass. The 1 mil thick sample data was chosen for analysis to minimize scattered neutron signal loss due to multiple scattering, where $1 \mathrm{mil}$ is 0.001 inches. The strong scattering resonances at $36 \mathrm{eV}, 66 \mathrm{eV}, 102 \mathrm{eV}$ and $189 \mathrm{eV}$ were analyzed.

The total resolution function is due to several factors, including the neutron burst width, the channel width, the target contribution, and the detector contribution ${ }^{9}$. Each component in the resolution function varies in importance, with the primary contribution coming from the additional path length to the detector for scattered neutrons at lower energies. At higher energies, the target contribution also becomes significant.

Accurate determination of the resolution shape for the target and detector are critical for the determination of the resonance parameters since all resolution effects broaden the experimental data and alter the resonance shape ${ }^{11}$. Currently the SAMMY inputs for channel width and burst width are valid, while the target moderator and detector shapes are not included. For scattered neutron analysis in SAMMY, these effects need to be included. MCNP modeling has determined the detector resolution shape due to the additional scattered neutron path lengths. These path lengths were parameterized to 
allow for use over the MCNP analyzed energy range. The scattering target resolution function was determined by comparison with experimental data using the method of Moretti ${ }^{9}$.

For the testing of the resolution shape, resonance data that is well documented and known with a high degree of confidence was chosen. This is the reason for using ${ }^{238} \mathrm{U}$ data. The chosen data is ${ }^{238} \mathrm{U}$ data from ENDF/B-VI Doppler broadened to $300 \mathrm{~K}$. The data are Doppler broadened to room temperature for reasons outlined by Moretti ${ }^{9}$.

\subsection{Broadening Process}

Both the total cross section and capture cross section data were downloaded from the Brookhaven National Laboratory to calculate scattering yield. These data were processed using a version of the capture analysis Mathcad programs, developed by Moretti ${ }^{9}$, which were altered for scattering analysis. The first alteration is the calculation of single-interaction scattering yield from ENDF data, which is shown in equation (4.2), where $\mathrm{E}$ is the energy, $\mathrm{n}$ is the sample number density of $1.3854 * 10^{-4}$ atom/barn, $\sigma_{\mathrm{tot}}$ is the total cross section, $\sigma_{\text {cap }}$ is the capture cross section, $Y_{\text {ths }}$ is the Doppler-broadened ENDF determined single-interaction scattering yield and $\mathrm{T}$ is the calculated transmission.

$$
\begin{gathered}
T(E)=\exp \left(-n \cdot \sigma_{t o t}(E)\right) \\
Y_{t h s}(E)=(1-T(E)) \cdot \frac{\sigma_{t o s}(E)-\sigma_{c a p}(E)}{\sigma_{t o t}(E)}
\end{gathered}
$$


The Doppler-broadened ENDF calculated yield is shown in figure 4.1 for energies of 30 to $115 \mathrm{eV}$.

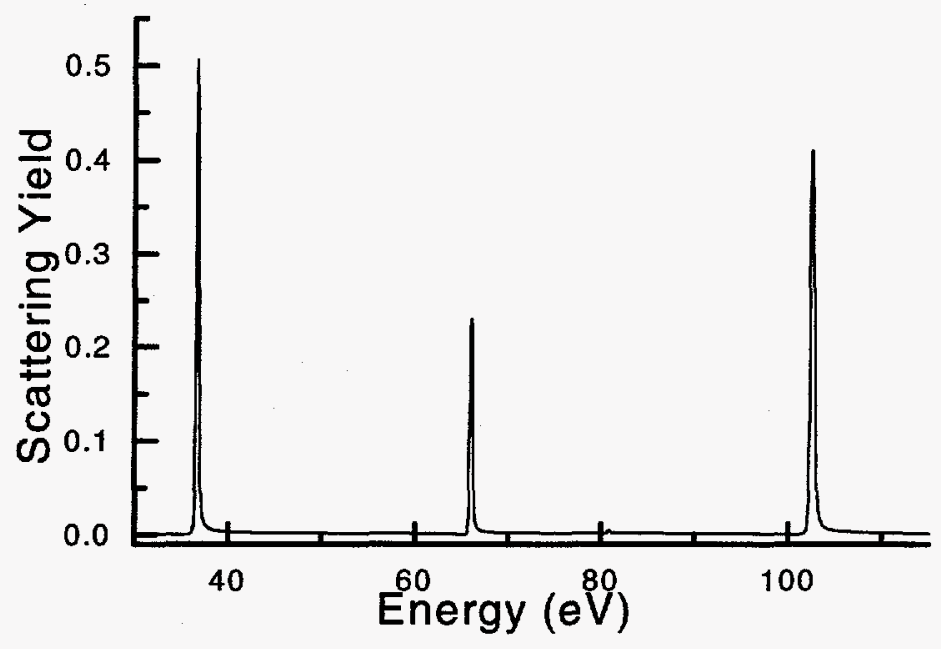

Figure 4.1 - Calculated yield from Doppler-broadened ENDF cross sections for the $1 \mathrm{mil}^{238} \mathrm{U}$ sample

The Doppler-broadened ENDF calculated yield were additionally broadened at all resonances with the channel width square function and the burst width gaussian function, which are the forms used by Moretti ${ }^{9}$. The resultant function was broadened by the calculated detector function and multiplied by an efficiency value that creates the best fit to the experimental data by having the lowest $\chi^{2}$. The starting point for the efficiency determination was a value in the range of the MCNP efficiency values listed in Table 2.1. Further broadening was performed to determine the target resolution function for neutron scattering through comparison of the broadened ENDF scattering yield and the experimental results. 


\subsection{Experimental Setup}

The data used for resolution function analysis comes from ${ }^{238} \mathrm{U}$ capture/scattering experiments on October $11-12,1995$. The samples and number of triggers for each sample are listed in table 4.1.

\begin{tabular}{|c|c|}
\hline Sample type and thickness & Triggers (x100) \\
\hline${ }^{238} \mathrm{U} 1$-mil & 2400 \\
\hline Empty can & 500 \\
\hline${ }^{238} \mathrm{U}$ 5-mil & 1000 \\
\hline Empty can & 100 \\
\hline${ }^{238} \mathrm{U} 10$-mil & 800 \\
\hline Empty can & 200 \\
\hline${ }^{238} \mathrm{U}$ 20-mil & 800 \\
\hline
\end{tabular}

Table 4.1 - Sample and number of triggers in capture/scatter experiment

For these experiments, the base channel width was set to $0.03125 \mu \mathrm{sec}$ and the LINAC burst width was $0.070 \mu \mathrm{sec}$. The data from the experiments were checked for statistical fluctuations, deadtime corrected, normalized and summed into a single file for each sample using the data processing programs on the HP-1000 computer for capture data.

\subsection{Experimental Data Processing}

Due to the radioactivity of the ${ }^{238} \mathrm{U}$ sample, background determination is not simple. The background was subtracted following the method of Moretti ${ }^{9}$. The empty can background was first subtracted on a channel-by-channel basis. The sample contribution to the background was then determined at each resonance through linear interpolation of the mean signal far out on the resonance wings. Errors were propagated throughout the 
process. The flux normalization factor was determined to be $13.60 \pm 0.16$ by following the method of Moretti ${ }^{9}$.

\subsection{Experimental Results}

The resolution function fits were determined for four resonances of the $1 \mathrm{mil}{ }^{238} \mathrm{U}$ sample to test the resolution function. Figure 4.2 below shows the result for the

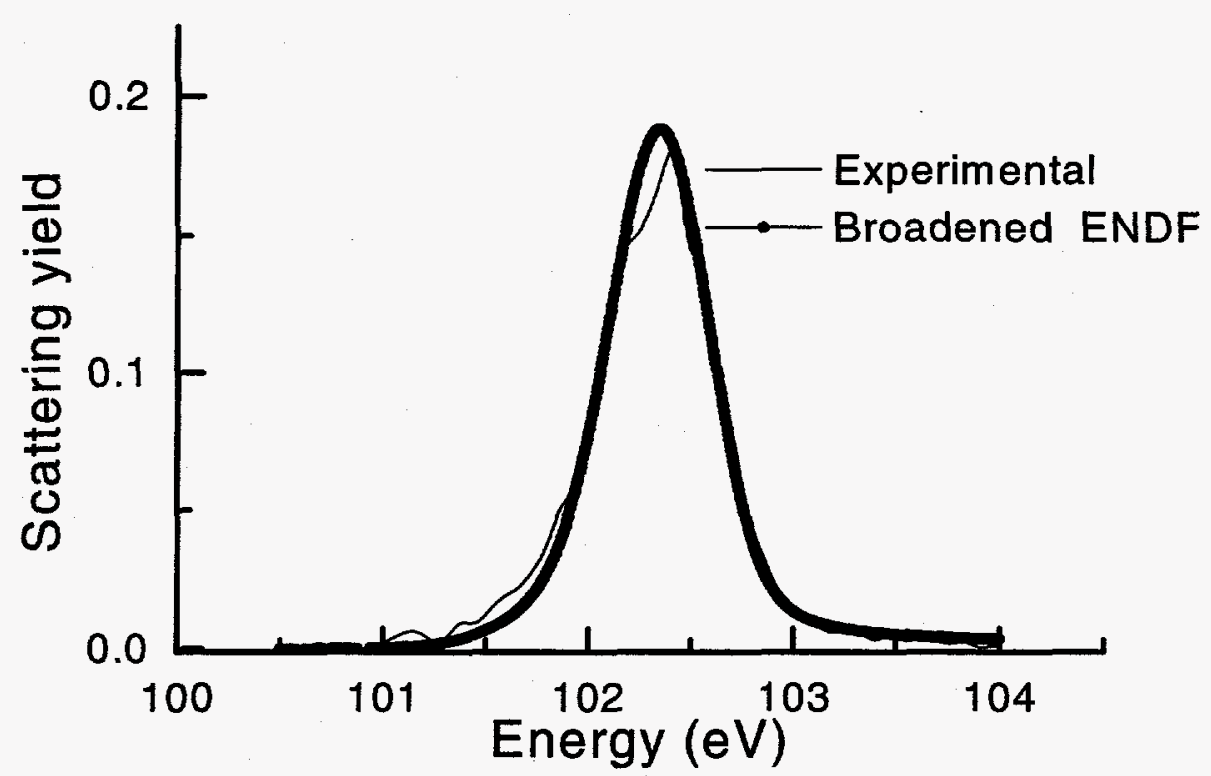

Figure 4.2 - Comparison of the resolution broadened ENDF calculated scattering yield and experimental scattering yield for the $102 \mathrm{eV}$ resonance for the $1 \mathrm{mil}^{238} \mathrm{U}$ sample

$102 \mathrm{eV}$ resonance of the $1 \mathrm{mil}{ }^{238} \mathrm{U}$ sample. The results for all of the resonances are shown in Appendix B. The ENDF calculated yield was burst width, channel width, and path length resolution broadened. For the $102 \mathrm{eV}$ resonance, the scattered neutron detection efficiency value of 0.562 was determined to have the minimum $\chi^{2}$ value of 5.93. The main portion of the curve is well represented by the resolution function. The 
peak and low energy tail regions are not as well represented. These two regions will be covered in detail in the next section. Table 4.2 below is a compilation of the scattered neutron efficiencies for the minimum $\chi^{2}$ along with the $\chi^{2}$ values for the compared resonances. Figure 4.3 shows the sensitivity of $\chi^{2}$ for the $102 \mathrm{eV}$ resonance.

\begin{tabular}{|c|c|c|}
\hline $\begin{array}{c}\text { Resonance } \\
\text { Energy }(\mathrm{eV})\end{array}$ & Detector Efficiency & Minimum $\chi^{2}$ \\
\hline 36.68 & 0.552 & 2.99 \\
\hline 66.03 & 0.580 & 2.34 \\
\hline 102.54 & 0.562 & 6.36 \\
\hline 189.7 & 0.560 & 8.24 \\
\hline
\end{tabular}

Table 4.2 - Resonance energy detector efficiency and fit values

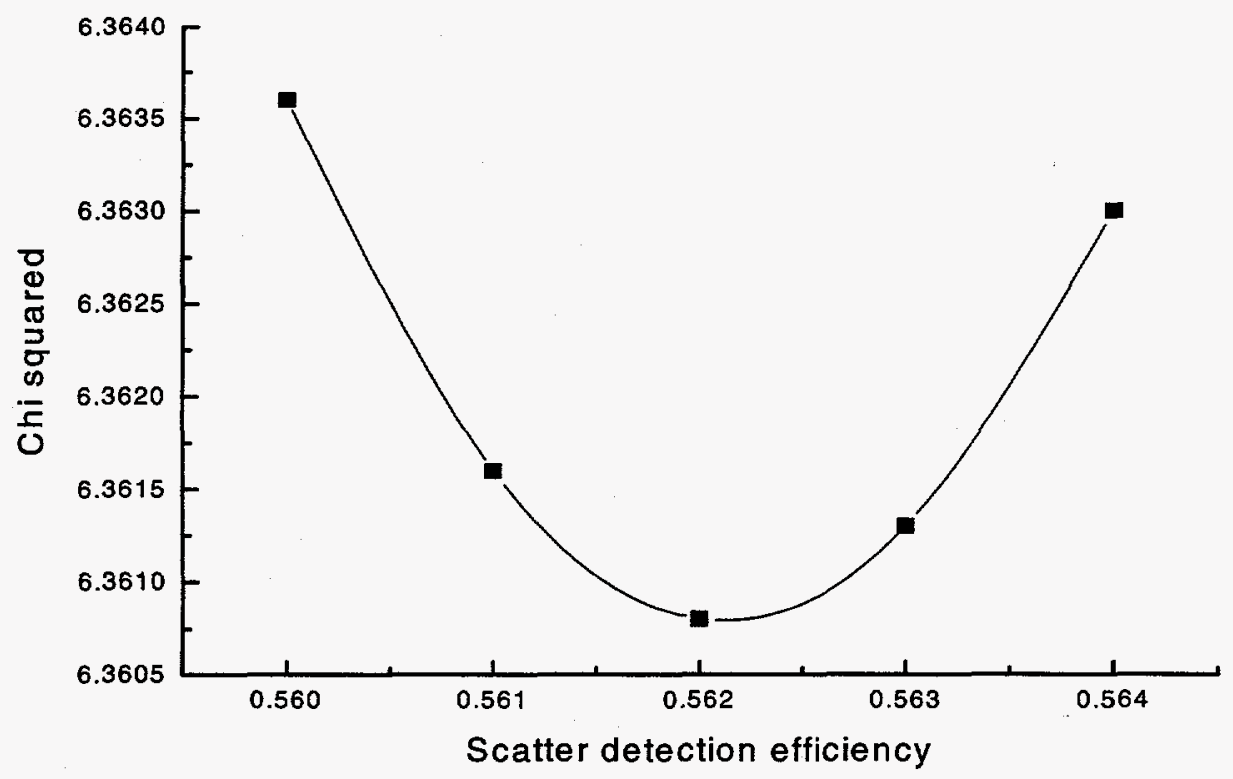

Figure 4.3 - Scatter detection efficiency sensitivity for the $102 \mathrm{eV}$ resonance for the $1 \mathrm{mil}^{238} \mathrm{U}$ sample 
From MCNP modeling, the expected detector efficiency is in the range of 0.55 to 0.575 between 10 and $100 \mathrm{eV}$. Also, as expected, the trend is decreasing efficiency as energy increases. The values in Table 4.2 for detector efficiency are between these two values, except for the $66 \mathrm{eV}$ resonance, which is approximately $1 \%$ higher than the maximum value. Overall, the detector efficiency remains a relatively constant value of 0.56 . The lower value determined at $36 \mathrm{eV}$ could be due to capture of scattered neutrons by the strong capture resonance. This will be covered further in Section 4.5.1.

\subsection{Discussion of Results}

\subsubsection{Peak Reduction}

The experimental resonance peak shown in figure 4.2 is distorted in shape. Similar effects were seen for all of the resonances, with the $36 \mathrm{eV}$ resonance having a flat top. This distortion could be due to capture of some of the scattered neutrons which would create a reduction of the scattering signal primarily at the peak.

Equation (4.1) was used to determine the fraction of the neutron beam which was transmitted through the sample. This value subtracted from 1 is the fraction of the beam interacting with the sample and is shown in Table 4.3 for the 1 mil ${ }^{238} \mathrm{U}$ sample for the Doppler broadened ENDF resonance peak total cross section values and an atomic density value of $1.385 * 10^{-4}$ atoms per barn. Multiple interactions are of concern when 


\begin{tabular}{|c|c|c|}
\hline Resonance energy $(\mathrm{eV})$ & ENDF $\sigma_{\text {tot }}($ barns $)$ & $\begin{array}{c}\text { Fraction of beam interacting with } \\
\text { sample }\end{array}$ \\
\hline 36.68 & $13,491.1$ & 0.846 \\
\hline 66.03 & 4387.4 & 0.445 \\
\hline 102.54 & 6068.0 & 0.568 \\
\hline 189.7 & 5224.7 & 0.515 \\
\hline
\end{tabular}

Table 4.3 - Fraction of beam interacting at ${ }^{238} \mathrm{U}$ resonances for 1 mil sample

more than approximately $10 \%$ of the beam interacts with the sample. This is certainly the case for the ${ }^{238} \mathrm{U}$ resonances of interest and should show up as a reduction of the scattering signal, primarily at the peak. This effect will have to be included for a complete scattering resolution function for thick samples.

\subsubsection{Lower Energy Wing Enhancement}

For each of the scattering resonances, the lower energy wing has a larger signal than that of the higher energy wing, while the Doppler-broadened ENDF curve has a lower energy wing signal smaller than that of the higher energy wing, shown in figure 4.1.

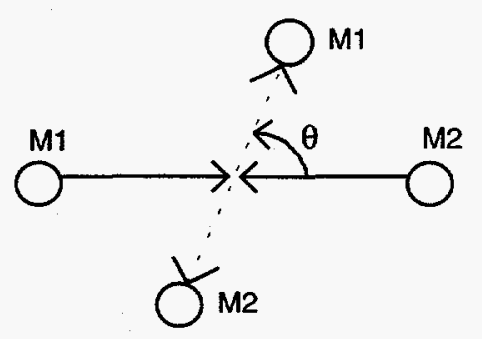

Figure 4.4 - Collision in the center-of-mass system where M1 is the incident particle 
The energy transferred to the ${ }^{238} U$ results in a lowering of the energy of thescattered neutron, which would result in an increase of the signal at the lower energy wing of the resonance at the expense of the neutrons with energies on the high energy side of the resonance. Figure 4.4 shows a collision in the center-of-mass (CM) system between an incoming mass $M_{1}$ and a target mass $M_{2}$ as well as the scattering angle $\theta$ in the $\mathrm{CM}$ system. The energy transferred from $\mathrm{M}_{1}$ to $\mathrm{M}_{2}$ is given by equation 4.3 where $\Lambda$ is defined in equation $4.4^{12}$. Equation (4.4) below shows the amount of energy

$$
\begin{gathered}
E_{\text {transfer }}(\theta)=\frac{1}{2} \Lambda E(1-\cos (\theta)) \\
\Lambda=\frac{4 M_{1} M_{2}}{\left(M_{1}+M_{2}\right)^{2}}
\end{gathered}
$$

transferred through elastic collisions ${ }^{12}$. The value of $E_{\text {transfer }}(\theta)$ is the function of energy transferred to $\mathrm{M}_{2}$ as a function of the scattering angle in the center-of-mass system. This has been determined by solving the equations of conservation of momentum and energy for the elastic collision between particles $M_{1}$ and $M_{2}$. The minimum value of $\theta=0^{\circ}$ represents forward scattering of $M_{1}$, while the maximum value of $\theta=180^{\circ}$ represents backward scattering of $\mathbf{M}_{1}$.

Calculations have been performed for collisions between a neutron and ${ }^{238} \mathrm{U}$ nucleus for neutrons scattering at angles of $0^{\circ}$ and $180^{\circ}$ in the center-of-mass system to determine the maximum effect on the measured energy. Equations (4.3), (4.5) and (4.6) were used for the calculation of expected measured neutron energy. The value used for

$$
E_{\text {scatter }}(\theta)=E-E_{\text {transfer }}(\theta)
$$




$$
E=\left(\frac{72.2997 \cdot 25.5784}{t-t_{0}}\right)^{2}
$$

$\mathrm{t}_{0}, 3.78562 \mu \mathrm{s}$, was determined from a gaussian fit to the gamma flash curve. The path length of the neutron down the flight tube is $25.5784 \mathrm{~m}$. The path length for the scattered neutrons is $16.814 \mathrm{~cm}$, which is the distance to the $\mathrm{B}_{4} \mathrm{C}$ liner inner surface directly below the ends of the detector and approximately the maximum path length traveled by a detected neutron. Equation (4.6) is the equation used to determine the experimentally measured energy of the neutron.

The results of the calculations are shown in Table 4.4. The size of the time bins for these experiments at this energy range is $0.0625 \mu$ s.

\begin{tabular}{|c|c|c|c|c|c|}
\hline $\begin{array}{c}\text { Incident } \\
\text { neutron } \\
\text { energy }(\mathrm{eV})\end{array}$ & $\begin{array}{c}\text { Scattering } \\
\text { angle, } \theta \\
(\text { degrees })\end{array}$ & $E_{\text {scatter }}(\theta)$ & $\begin{array}{c}\text { Time shift } \\
(\mu \mathrm{s})\end{array}$ & $\begin{array}{c}\text { Experimental } \\
\text { determined } \\
\text { energy }(\mathrm{eV})\end{array}$ & $\begin{array}{c}\text { Maximum } \\
\text { energy shift } \\
(\mathrm{eV})\end{array}$ \\
\hline 36.68 & & & & & 0.0004 \\
\hline & 0 & 36.68 & 2.007 & 36.203 & \\
\hline & 180 & 36.06 & 2.024 & 36.199 & \\
\hline 66.03 & & & & & 0.008 \\
\hline & 0 & 66.03 & 1.496 & 65.171 & \\
\hline & 180 & 64.91 & 1.509 & 65.163 & \\
\hline 102.2 & & & & & 0.012 \\
\hline & 0 & 102.2 & 1.202 & 100.870 & \\
\hline & 180 & 100.5 & 1.213 & 100.858 & \\
\hline 189.7 & & & & & 0.02 \\
\hline & 0 & 189.7 & 0.883 & 187.23 & \\
\hline & 180 & 186.5 & 0.890 & 187.21 & \\
\hline
\end{tabular}

Table 4.4 - Calculated measured energy shift due to nuclear recoil for ${ }^{238} \mathrm{U}$ 
Recoil calculations were performed for the lighter metals of elemental Molybdenum ( $\mathrm{M}=95.94 \mathrm{AMU})$ and Iron $(\mathrm{M}=55.847 \mathrm{AMU})$. Tables 4.5 and 4.6 display the results. In all cases, the experimental energy shift due to recoil of the target nucleus is negligible.

\begin{tabular}{|c|c|c|c|c|c|}
\hline $\begin{array}{c}\text { Incident } \\
\text { neutron } \\
\text { energy }(\mathrm{eV})\end{array}$ & $\begin{array}{c}\text { Scattering } \\
\text { angle, } \theta \\
(\text { degrees })\end{array}$ & $E_{\text {scatter }}(\theta)$ & $\begin{array}{c}\text { Time shift } \\
(\mu \mathrm{s})\end{array}$ & $\begin{array}{c}\text { Experimental } \\
\text { determined } \\
\text { energy }(\mathrm{eV})\end{array}$ & $\begin{array}{c}\text { Maximum } \\
\text { energy shift } \\
(\mathrm{eV})\end{array}$ \\
\hline 20 & & & & & 0.006 \\
\hline & 0 & 20.00 & 2.718 & 20.478 & \\
\hline & 180 & 19.18 & 2.775 & 20.472 & \\
\hline 200 & & & & & 0.054 \\
\hline & 0 & 200.00 & 0.860 & 197.394 & \\
\hline & 180 & 191.83 & 0.878 & 197.340 & \\
\hline
\end{tabular}

Table 4.5 - Calculated measured energy shift due to nuclear recoil for Mo

\begin{tabular}{|c|c|c|c|c|c|}
\hline $\begin{array}{c}\text { Incident } \\
\text { neutron } \\
\text { energy }(\mathrm{eV})\end{array}$ & $\begin{array}{c}\text { Scattering } \\
\text { angle, } \theta \\
(\text { degrees })\end{array}$ & $E_{\text {scatter }}(\theta)$ & $\begin{array}{c}\text { Time shift } \\
(\mu \mathrm{s})\end{array}$ & $\begin{array}{c}\text { Experimental } \\
\text { determined } \\
\text { energy }(\mathrm{eV})\end{array}$ & $\begin{array}{c}\text { Maximum } \\
\text { energy shift } \\
(\mathrm{eV})\end{array}$ \\
\hline 20 & & & & & 0.010 \\
\hline & 0 & 20.00 & 2.718 & 20.478 & \\
\hline & 180 & 18.62 & 2.817 & 20.468 & \\
\hline 200 & & & & & 0.093 \\
\hline & 0 & 200.00 & 0.860 & 197.394 & \\
\hline & 180 & 186.18 & 0.891 & 197.301 & \\
\hline
\end{tabular}

Table 4.6 - Calculated measured energy shift due to nuclear recoil for $\mathrm{Fe}$

Table 4.4 demonstrates that the approximation of the ${ }^{238} \mathrm{U}$ nucleus having an infinite mass is acceptable, since the maximum time shift due to recoil energy loss is much less than the size of the time bins used in the experiments. The recoil calculations 
performed for Molybdenum and Iron show that the maximum recoil losses for the lower energy neutrons can result in a time shift equal to or greater than one channel width. This can distort the experimental data. Nuclear recoil effects will have to be taken into account for scattering measurements of samples with lighter nuclei.

The previous calculations have not taken into account the delay in detection of scattered neutrons resulting from the path length traveled by the photons created in the detector liner prior to detection. Using dimensions from the detector model of figure 2.2 , the maximum path length traveled by a photon prior to detection is determined to be $40.28 \mathrm{~cm}$. Since the photon travels at the speed of light, the resultant maximum time shift is $1.34 \times 10^{-9}$ seconds. This shift is too small to be detected, which justifies the neglecting of the detection path length of the photons created in the liner.

\subsubsection{Scattering Target Resolution Function}

Only the burst width, channel width and detector resolution functions were considered. The fits for the lower energy scattering resonances are reasonably good, while the fits degrade at resonance energy values greater than $100 \mathrm{eV}$. Since nuclear recoil energy and photon path length effects are minimal, this must be due primarily to the target resolution.

To determine the target resolution, the effects of the other three factors have to be known to a high degree of confidence. Both channel width and burst width effects are previously known ${ }^{9}$. The scattering detector resolution function determined earlier is reasonably well-known for the following reasons. First, the time distribution of neutron 
intensity on the inner liner surface resulting from a monoenergetic neutron point source matches the shape of the analytical solution. Second, the MCNP generated curves for the time distribution of photon intensity for the monoenergetic 2 -inch disk source are similar in path length space and show the additional path length traveled into the liner by the higher energy neutrons. Third, the shifting of the resonance peaks is accurately determined by the MCNP model, as shown in the curves in Appendix B. Fourth, the detector efficiency determined from MCNP energy deposition calculations is in good agreement with the values that result in the best correlation between the broadened ENDF calculated scattering yield and the experimental yield. Fifth, the fits for the lower energy resonances are already reasonably close.

The starting point for the scattering target resolution function is the capture resolution function determined by Moretti for the bounce target ${ }^{9}$. The following chisquare plus sum of exponentials function from Moretti was used to generate the target resolution function, $R(t)$, as a function of time:

$$
R(t)=\frac{(t+\text { shiff })^{2}}{2 \cdot \Lambda^{3}} \exp \left(\frac{-(t+\text { shift })}{\Lambda}\right)+A_{1}\left(A_{2} \exp \left(A_{3}(t+0.94)+A_{4} \exp \left(A_{5}(t+0.94)\right)\right)\right.
$$

The value for ' $\Lambda$ ' as a function of energy was determined from the following equation:

$$
\Lambda(E)=0.3017-0.08739 \cdot \ln (E)+0.00706 \cdot(\ln (E))^{2}
$$

The value for 'shift' as a function of energy was determined from the following equation:

$$
\operatorname{shift}(E)=0.381 \cdot \exp (-0.019 \cdot E)+0.133 \cdot \exp (-0.094 \cdot E)+0.105
$$

The value for ' $\mathrm{A}_{1}$ ' as a function of energy was determined from the following equation:

$$
A_{1}(E)=-1.106 \cdot \exp (-0.0058 \cdot E)+47.52 \cdot \exp (-65.083 \cdot E)+1.264
$$


The values used for the constants ' $\mathrm{A}_{2}$ ' through ' $\mathrm{A}_{5}$ ' are listed in Table 4.7.

\begin{tabular}{|c|c|c|c|}
\hline $\mathrm{A}_{2}$ & $\mathrm{~A}_{3}$ & $\mathrm{~A}_{4}$ & $\mathrm{~A}_{5}$ \\
\hline-65.64 & -5 & 0.3898 & -0.8 \\
\hline
\end{tabular}

Table 4.7 - Constants used in capture and scattering target resolution functions

The resultant resolution function is shown below in Figure 4.5 for a neutron energy of $189.7 \mathrm{eV}$.

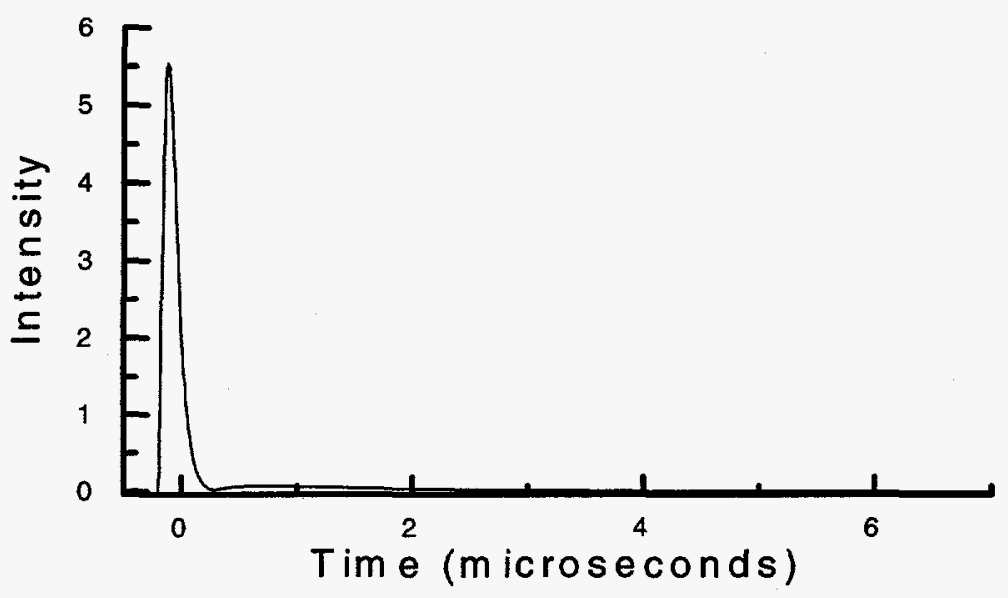

Figure 4.5 - Capture target resolution function at $189.7 \mathrm{eV}$

The application of this resolution function to the previously broadened calculated ENDF scattering yield results in a resonance curve which is both exceedingly broadened and shifted toward higher energy. The reason for the shift is yet to be determined. The excessive broadening of the calculated resonance curve is the result of the sum of exponentials tail being too large in magnitude. Also, the curve has a secondary peak due to the sum of exponentials tail. The tail has been shifted to make the resolution function smooth. Both the shift of the chi-square function and the magnitude of the tail have also 
been adjusted to create good agreement between the experimental data and the broadened ENDF parameters.

The resulting fit for the $189.7 \mathrm{eV}$ resonance using the modified scattering target resolution function is shown in Figure 4.6. The fits for all of the resonances are

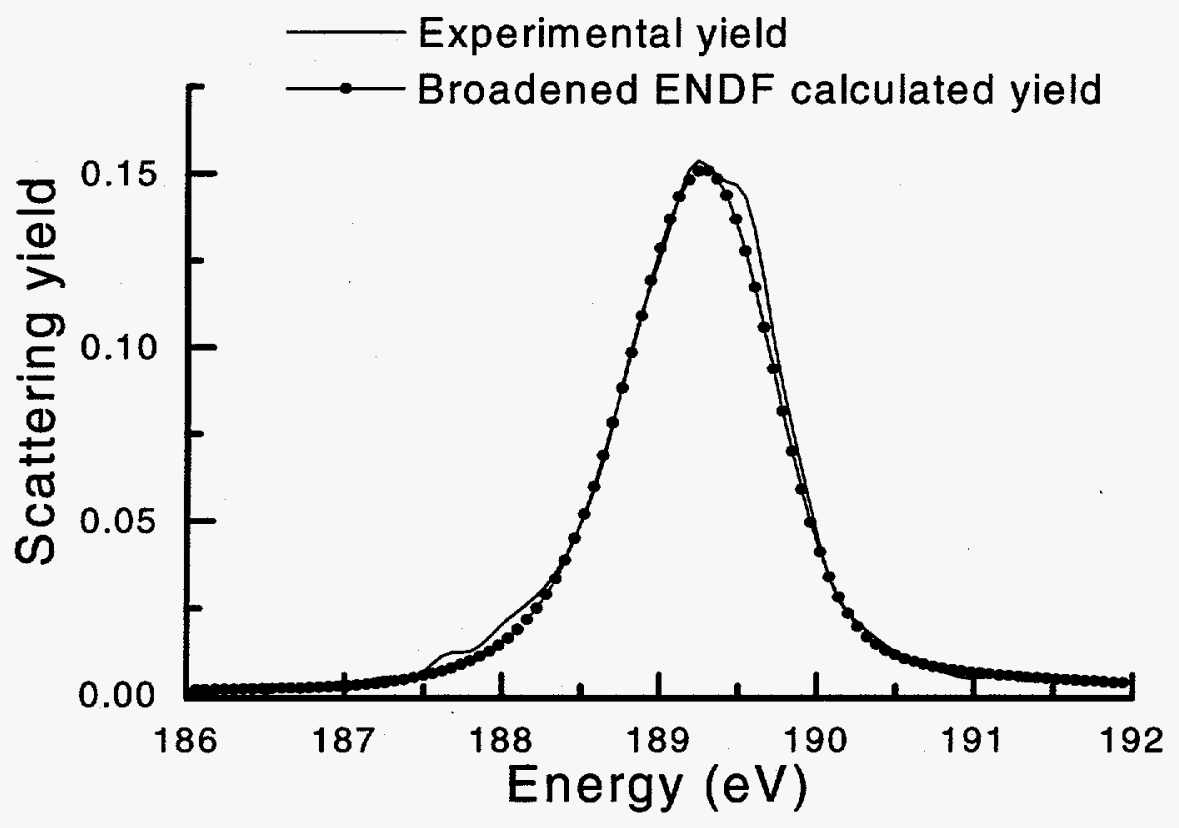

Figure 4.6 - Experimental yield versus broadened ENDF calculated yield for the ${ }^{238} \mathrm{U} 189.7 \mathrm{eV}$ resonance

displayed in Appendix C. Table 4.8 is a listing of the adjusted parameters and the goodness-of-fit values for all of the resonances.

\begin{tabular}{|c|c|c|c|}
\hline Energy $(\mathbf{e V})$ & shift & $\mathbf{A}_{\mathbf{1}}$ & $\boldsymbol{\chi}^{\mathbf{2}}$ \\
\hline $\mathbf{3 6 . 6 8}$ & 0.223 & 0.064 & 1.796 \\
\hline $\mathbf{6 6 . 0 3}$ & 0.185 & 0.116 & 1.384 \\
\hline $\mathbf{1 0 2 . 2}$ & 0.155 & 0.180 & 2.617 \\
\hline $\mathbf{1 8 9 . 7}$ & 0.120 & 0.333 & 2.653 \\
\hline
\end{tabular}

Table 4.8 - Modified target results 
The modified target resolution function for the $189.7 \mathrm{eV}$ resonance is shown in Figure 4.7

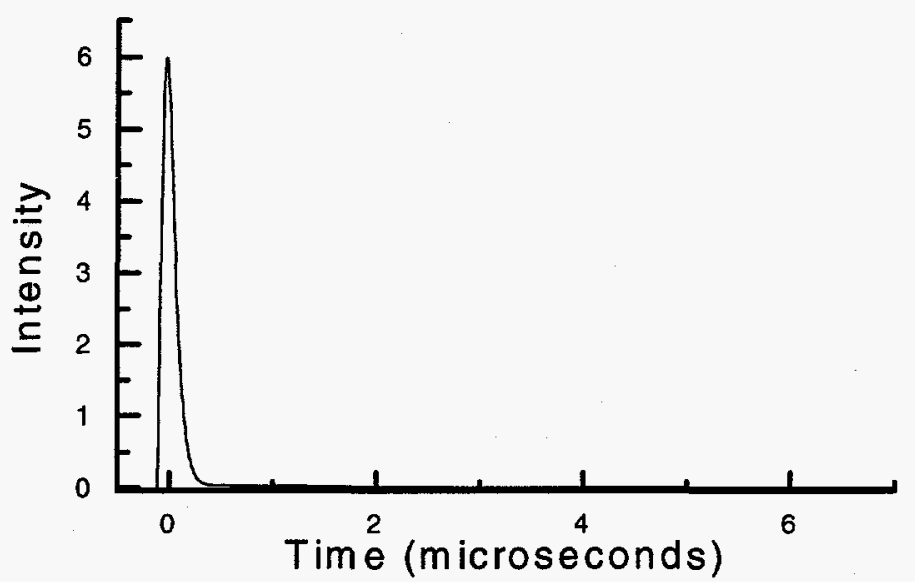

Figure 4.7 - Scattering target resolution function for $189.7 \mathrm{eV}$

The change to the resolution equation of (4.7) is the constant that shifts the peak of the exponentials. Equation (4.11) with the additional shift replaces equation (4.7).

$$
R(t)=\frac{(t+\text { shift })^{2}}{2 \cdot \Lambda^{3}} \exp \left(\frac{-(t+\text { shift })}{\Lambda}\right)+A_{1}\left(A_{2} \exp \left(A_{3}(t+1.207)+A_{4} \exp \left(A_{5}(t+1.207)\right)\right)\right.
$$

The values for 'shift' have been fitted by equation (4.12), which replaces equation (4.9).

$$
\operatorname{shift}(E)=0.52419-0.0979 \cdot \ln (E)+0.00394 \cdot(\ln (E))^{2}
$$

Since the magnitude of the tail is small, equation (4.13) has been used to replace equation (4.10). Further refinement of the relationship is possible, although this simple relationship provides good results for energies up to $190 \mathrm{eV}$.

$$
A_{1}(E)=\frac{0.3333 \cdot E}{189.7}
$$

These modifications to the previously determined capture resolution functions provide excellent matches to the ${ }^{238} \mathrm{U}$ experimental scattering data over the analyzed 
energy range. Currently, there is no explanation for the difficulties experienced with the previously determined resolution functions, although the modifications do work. The neutron tail is smaller than the previously determined tail. Possibly this is due to the decoupling of the target and detector resolution effects. 


\section{CONCLUSIONS}

This thesis presents a resolution function applicable to low energy neutron scattering measurements performed at RPI. This resolution function has been parameterized both in neutron energy and sample diameter. Scattered neutron detection efficiency has also been determined.

The experimentally determined scattering resonance has a peak which is lower in energy and broader than the corresponding capture resonance peak. This is primarily due to the additional and varied distance traveled by the scattered neutrons prior to detection. MCNP models were used to determine the distribution of the additional path lengths traveled by the scattered neutrons prior to detection. These models used isotropic sources, which simulate s-wave neutron scattering with nuclear recoil neglected. The time distribution of neutron current entering the inner surface of the $\mathrm{B}_{4} \mathrm{C}$ liner and photon current exiting the outer surface of the $\mathrm{B}_{4} \mathrm{C}$ liner were determined in this manner.

The simplest model of the monoenergetic neutron source is the isotropic point source. The MCNP results are in good agreement with the analytical solution.

The next series of MCNP models consist of monoenergetic isotropic disk sources of varying size and energy. For the varying energy, the size was fixed at the standard RPI sample diameter of 2 inches. For the varying size, the energy was fixed at $10 \mathrm{eV}$. The resulting curves were successfully reproduced and parameterized using an asymmetric double cumulative curve combined with a sum of exponentials tail. 
The energy parameterized curves were tested against experimental uranium scattering measurements. The lower energy curves were well reproduced. However, the fit degraded at higher energies due to the scattering target resolution.

The scattering target resolution function was determined for the ${ }^{238} \mathrm{U}$ resonances up to $187.9 \mathrm{eV}$. This function is a modified form of the capture target resolution function for the bounce target. The modifications produced an excellent fit to the experimental data.

There are additional potential improvements to the scattering resolution function which were not addressed in this thesis. For example, nuclear recoil effects could be taken into account to allow for determination of scattering resonance parameters for lighter nuclei samples, the scattering target resolution could be improved, and the scattering resolution function may also be tested against different materials to ensure further applicability. 


\section{REFERENCES}

1. N. M. Larson, SAMMY-H1: A Code System for Multilevel R-Matrix Fits to Neutron Data Using Bayes Equations, ORNL/TM-9179/R2 \& Draft R3 (1994).

2. R. E. Slovacek, R. C. Block, Y. Danon, C. Werner, G. Youk, J. A. Burke, N. J

Drindak, F. Feiner, J. A. Helm, K. W. Seemann, "Neutron Cross Section Measurements at the Rensselaer LINAC", Proceedings of the Topical Conference on Advances in Reactor Physics, Knoxville, TN (April, 1994).

3. MCNP, Monte Carlo N-Particle Transport Code System, Version 4A, Los Alamos National Laboratory, LA-12625-M (1993).

4. G. Leinweber, Calculations of Multiplicity and Efficiency from Neutron Capture in the RPI Multiplicity Detector, Doctoral Thesis, Rensselaer Polytechnic Institute (1994).

5. L. I. Maissel, R. Glang, Handbook of Thin Film Technology, McGraw-Hill (1970).

6. TableCurve 2D Windows V2.0, AISN Software (1994).

7. W. H. Beyer, CRC Standard Mathematical Tables $28^{\text {th }}$ Edition, CRC Press (1987).

8. Microcal Origin Version 4.00, Microcal Software (1995).

9. B. E. Moretti, Molybdenum Neutron Transmission Measurements and the Development of an Enhanced Resolution Neutron Target, Doctoral Thesis, Rensselaer Polytechnic Institute (1996).

10. Mathcad version 6.0 Plus, MathSoft (1995). 
11. D. K. Olsen, G. de Saussure, R. B. Perez, F. C. Difilippo, R. W. Engle, H. Weaver, "Measurement and Resonance Analysis of Neutron Transmission Through Uranium238", Nuclear Science and Engineering, 69, 202-222 (1979).

12. R. D. Evans, The Atomic Nucleus, McGraw-Hill, (1955). 
APPENDIX A

\section{SAMPLE MCNP MODELING INPUT FILES}

A.1 - Sample MCNP input file for disk source neutron current on inner liner surface

2.0 inch diameter $10 \mathrm{eV}$ neutron photon problem

c

c cells:

c

$\begin{array}{lllllllll}1 & 1 & -2.31 & 3 & -4 & 1 & -2 & \$ \mathrm{~b} 4 \mathrm{c} \text { liner }\end{array}$

$\begin{array}{llllll}2 & 0 & -1 & 3 & -4 & \text { \$ inner void }\end{array}$

$\begin{array}{llllll}3 & 0 & 2 & -3 & 4 & \text { \$ outside world }\end{array}$

c surfaces:

c

$1 \mathrm{cz} 3.18$

$2 . \mathrm{cz} 4.18$

$3 \mathrm{pz}-1.515$

$\begin{array}{lll}4 & \mathrm{pz} & 34.535\end{array}$

mode $\mathrm{n} \mathrm{p}$

sdef $\operatorname{cel}=2$ erg=1.0e-5 pos $=0016.4973 \mathrm{rad}=\mathrm{d} 1 \mathrm{axs}=001 \mathrm{ext}=0$

si1 $\quad 02.54127$

imp:n 1110

imp:p 110

m1 $50100.7956850110 .004436012 .19786013 .0022 \$$ b4c

f1:p 2

e1 $\quad .36 \quad 68 \quad 100$

t1 182021.422 .8242526272829303132333435363738394041 4243444546474849505152535455565758596061626364

656667686970717273747576777879

80818283848586878889909192939495969798

99100101102103104105106107108109110111112113114

115116117118119120121122123124125126128130132134

136138140142144146148150154158162166170174

$1781861952072132272412602903851 \mathrm{E} 33$

nps 1000000

print 


\section{A.2 - Sample MCNP input file for energy deposition determination}

sodium iodide detector $w / b 4 c$ liner efficiency

$\mathrm{c}$

c cells:

c

$\begin{array}{lllllllll}1 & 3 & -3.67 & 18 & -17 & 9 & -10 & 5 & -6\end{array}$

$\begin{array}{lllllllll}2 & 3 & -3.67 & 17 & -16 & 9 & -10 & 5 & -6\end{array}$

$\begin{array}{lllllllll}3 & 3 & -3.67 & 16 & 19 & 9 & -10 & 5 & -6\end{array}$

$\begin{array}{lllllllll}4 & 3 & -3.67 & 18 & -19 & 9 & -10 & 5 & -6\end{array}$

$\begin{array}{lllllllll}5 & 3 & -3.67 & 17 & -18 & 9 & -10 & 5 & -6\end{array}$

$\begin{array}{lllllllll}6 & 3 & -3.67 & 16 & -17 & 9 & -10 & 5 & -6\end{array}$

$\begin{array}{lllllllll}7 & 3 & -3.67 & -16 & -19 & 9 & -10 & 5 & -6\end{array}$

$\begin{array}{lllllllll}8 & 3 & -3.67 & 19 & -18 & 9 & -10 & 5 & -6\end{array}$

$\begin{array}{lllllllll}9 & 3 & -3.67 & 18 & -17 & 13 & -14 & 5 & -6\end{array}$

$\begin{array}{lllllllll}10 & 3 & -3.67 & 17 & -16 & 13 & -14 & 5 & -6\end{array}$

$\begin{array}{lllllllll}11 & 3 & -3.67 & 16 & 19 & 13 & -14 & 5 & -6\end{array}$

$\begin{array}{lllllllll}12 & 3 & -3.67 & 18 & -19 & 13 & -14 & 5 & -6\end{array}$

$\begin{array}{lllllllll}13 & 3 & -3.67 & 17 & -18 & 13 & -14 & 5 & -6\end{array}$

$\begin{array}{lllllllll}14 & 3 & -3.67 & 16 & -17 & 13 & -14 & 5 & -6\end{array}$

$\begin{array}{lllllllll}15 & 3 & -3.67 & -16 & -19 & 13 & -14 & 5 & -6\end{array}$

$\begin{array}{lllllllll}16 & 3 & -3.67 & 19 & -18 & 13 & -14 & 5 & -6\end{array}$

$\begin{array}{lllllll}17 & 1 & -2.70 & 8 & -31 & 4 & -30\end{array}$

$\begin{array}{lllllll}18 & 1 & -2.70 & 32 & -33 & 4 & -30\end{array}$

$\begin{array}{llllllll}19 & 2 & -2.31 & 35 & -36 & 1 & -2\end{array}$

$\begin{array}{llllll}20 & 2 & 8 & -15 & 2 & -4\end{array}$

$\begin{array}{llllll}21 & 4 & -2.51 & 11 & -12 & -20\end{array}$

$\begin{array}{lllllll}22 & 5 & -2.50 & 6 & -30 & 9 & -32\end{array}$

$\begin{array}{llllllll}23 & 1 & -2.70 & 34 & -15 & 4 & -30\end{array}$

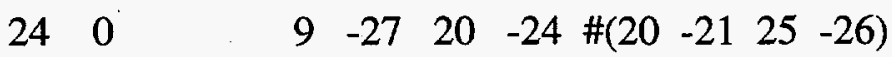

$\begin{array}{rrrrrrr}25 & 5 & -2.50 & 6 & -30 & 9 & -32\end{array}$

$\begin{array}{lllllll}26 & 1 & -2.70 & -15 & 8 & -7 & 30\end{array}$

$\begin{array}{lllll}27 & 0 & 35 & -9 & -1\end{array}$

$\begin{array}{llllll}28 & 0 & 9 & -36 & -1 & 24\end{array}$

$29 \quad 0 \quad \begin{array}{lllllll}-36 & -23 & 12 & \#(26-28+22-23) \#(29-23-26 & 12)\end{array}$

$\begin{array}{lllllll}30 & 0 & 9 & -11 & -20\end{array} \quad \#\left(\begin{array}{lllll}-11 & 25 & 29 & -20\end{array}\right)$

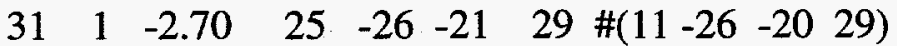

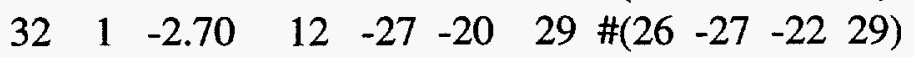

$\begin{array}{lllllll}33 & 1 & -2.70 & 27 & -28 & 22 & -24\end{array}$

$\begin{array}{lllllll}34 & 1 & -2.70 & 28 & -36 & 23 & -24\end{array}$

$\begin{array}{lllllll}35 & 5 & -2.50 & 31 & -9 & 4 & -30\end{array}$

$\begin{array}{lllllll}36 & 5 & -2.50 & 10 & -32 & 5 & -6\end{array}$

$\begin{array}{lllllll}37 & 5 & -2.50 & 33 & -13 & 5 & -6\end{array}$ 


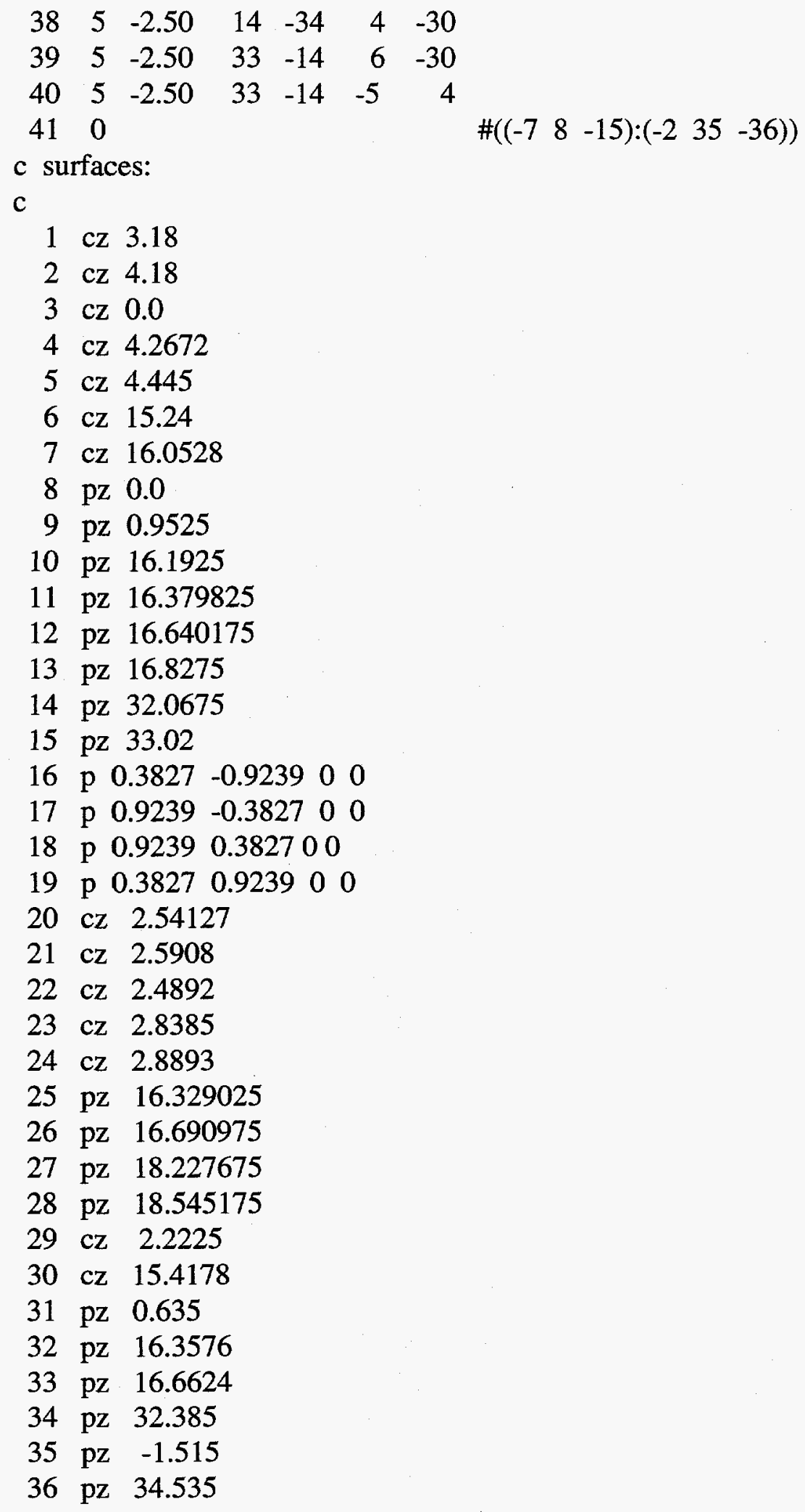




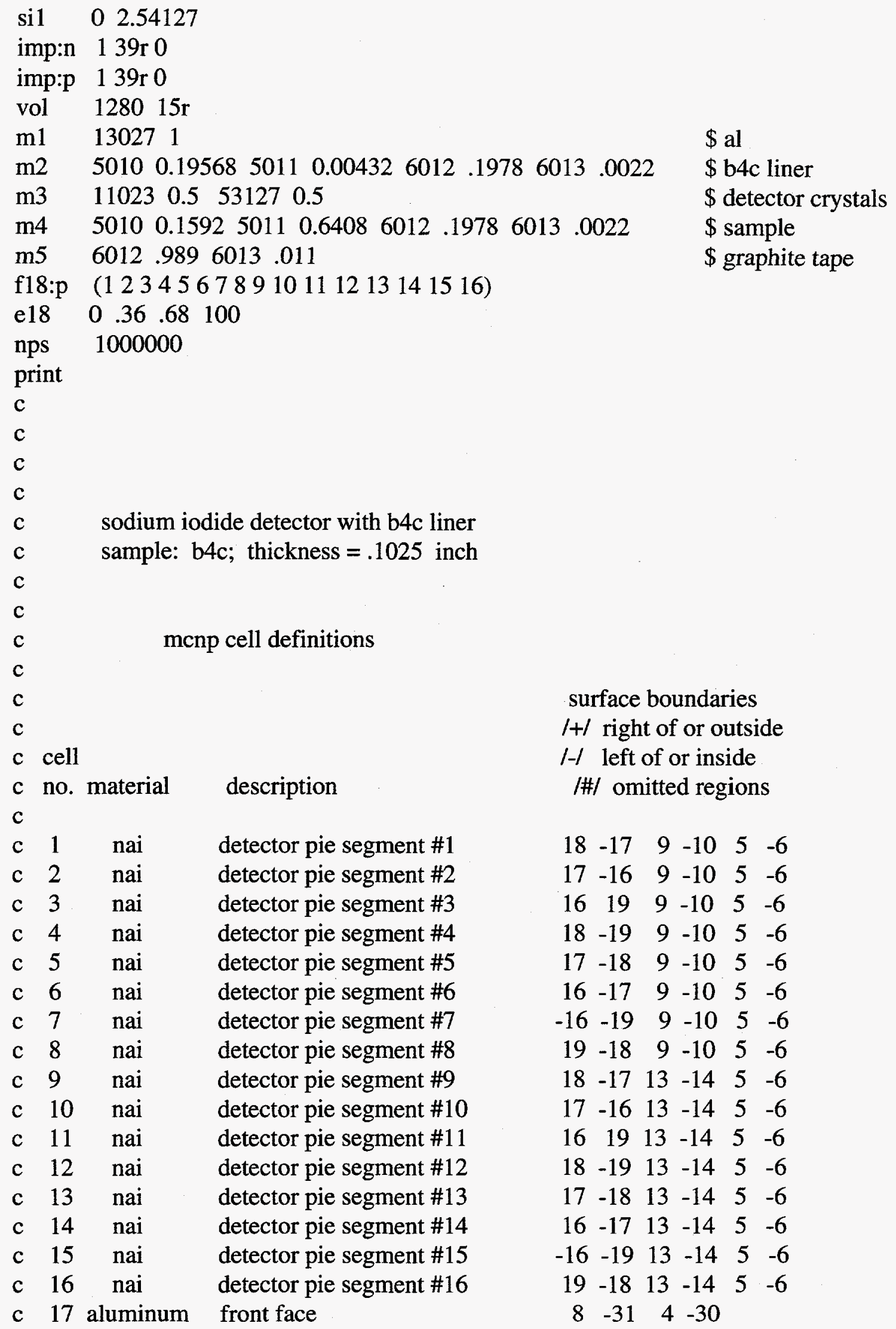




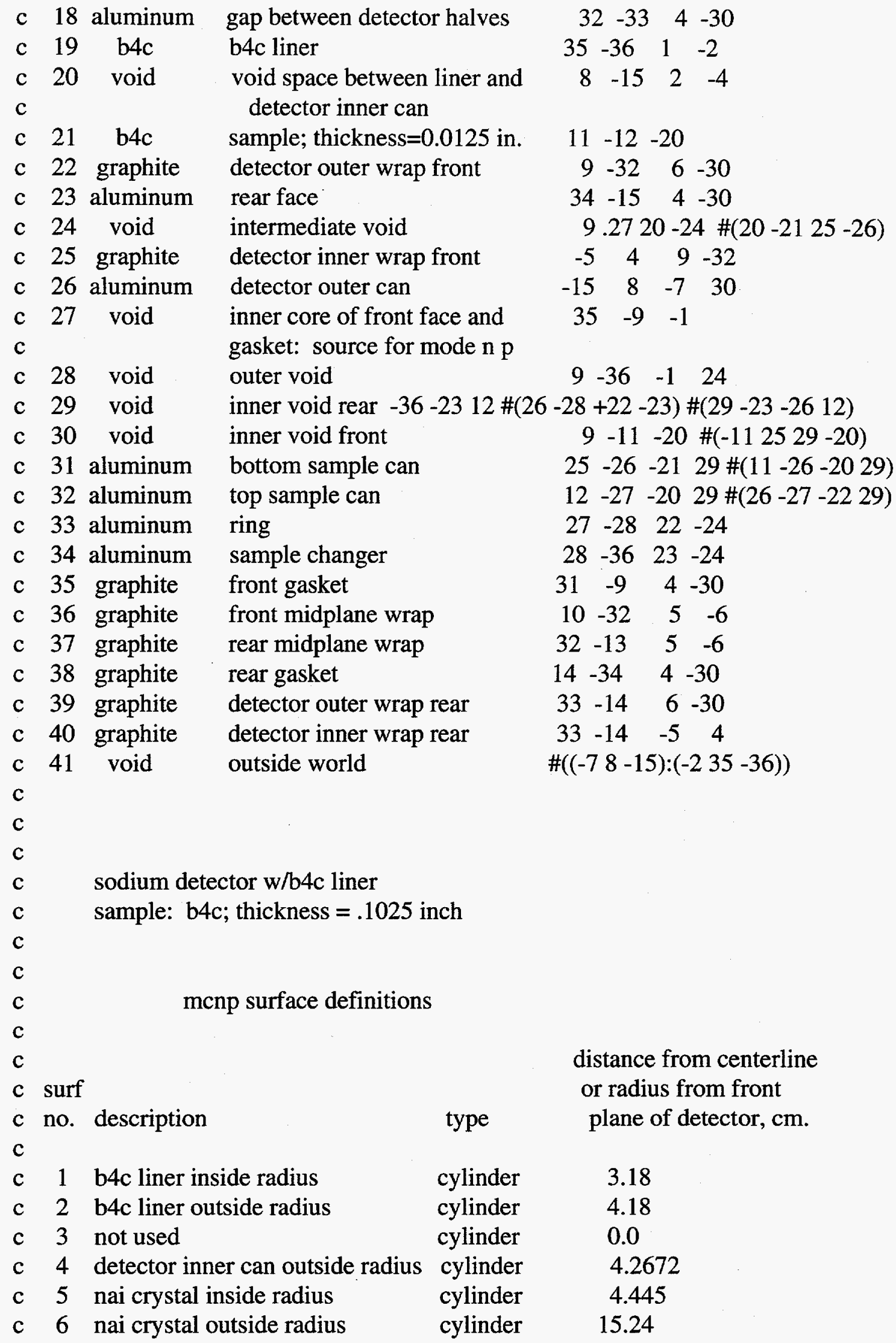




\begin{tabular}{|c|c|c|c|c|}
\hline c & 7 & total outside radius & cylinder & 16.0528 \\
\hline c & 8 & front face front & plane & 0.0 \\
\hline $\mathrm{c}$ & 9 & front crystal front & plane & 0.9525 \\
\hline c & 10 & front midplane wrap front & plane & 16.1925 \\
\hline c & 11 & front sample plane & plane & 16.379825 \\
\hline$c$ & 12 & rear sample plane & plane & 16.640175 \\
\hline c & 13 & rear midplane wrap rear & plane & 16.8275 \\
\hline c & & rear crystal front & & \\
\hline c & 14 & rear crystal rear & plane & 32.0675 \\
\hline c & 15 & end detector plane & plane & 33.02 \\
\hline c & 16 & diag plane to divide pie segments & plane & diagonal \\
\hline c & 17 & diag plane to divide pie segments & plane & diagonal \\
\hline c & 18 & diag plane to divide pie segments & plane & diagonal \\
\hline c & 19 & diag plane to divide pie segments & plane & diagonal \\
\hline c & 20 & sample radius & cylinder & 2.54127 \\
\hline c & 21 & bottom sample can outside radius & cylinder & 2.5908 \\
\hline c & 22 & ring inside radius & cylinder & 2.4892 \\
\hline$c$ & 23 & sample changer inside radius & cylinder & 2.8385 \\
\hline c & 24 & sample changer outside radius & cylinder & 2.8893 \\
\hline c & 25 & bottom sample can front plane & plane & 16.329025 \\
\hline c & 26 & top sample can rear plane & plane & 16.690975 \\
\hline c & 27 & front ring plane & plane & 18.227675 \\
\hline c & 28 & sample changer front plane & plane & 18.545175 \\
\hline $\mathrm{c}$ & 29 & sample can hole radius & cylinder & 2.2225 \\
\hline c & 30 & outside wrap outside radius & cylinder & 15.4178 \\
\hline c & 31 & front gasket front & plane & 0.635 \\
\hline c & 32 & gap between detector halves front & plane & 16.3576 \\
\hline c & 33 & gap between detector halves rear & plane & 16.6624 \\
\hline c & 34 & rear gasket rear & plane & 32.385 \\
\hline c & 35 & $\mathrm{~b} 4 \mathrm{c}$ liner front & plane & -1.515 \\
\hline c & 36 & b4c liner rear & plane & 34.535 \\
\hline
\end{tabular}




\section{APPENDIX B ${ }^{238}$ U RESONANCE FITS FOR DETECTOR RESOLUTION}

Bounce target data: Resolution fit to 1 mil Uranium sample at $36 \mathrm{eV}$ Resonance

\section{SCATTER DATA}

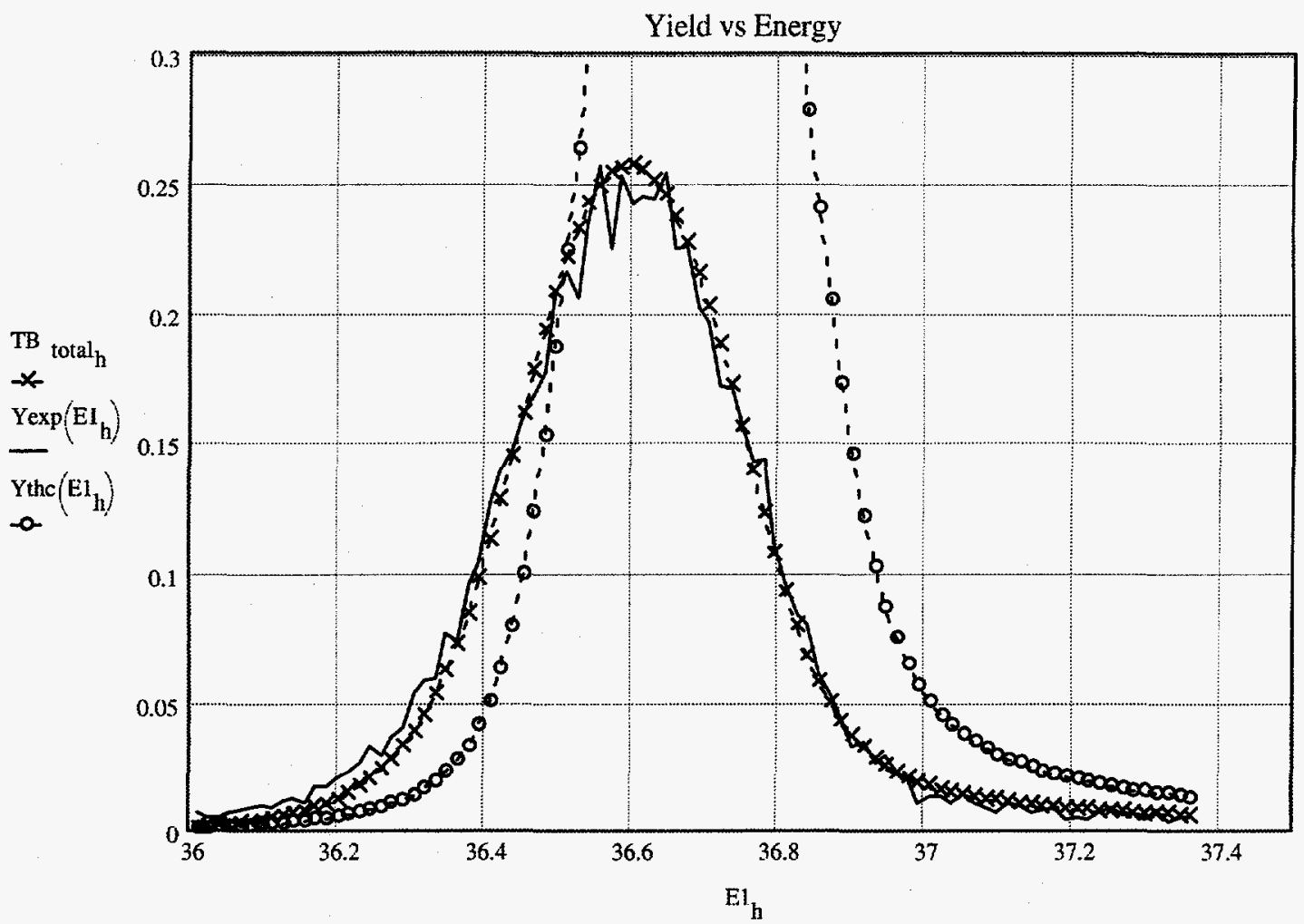

TBtotal: Scattering yield calculated from Doppler broadened ENDF cross sections using a combined resolution function consisting of channel width, burst width and detector functions. Detector efficiency used is 0.552 .

Yexp: Experimental scattering yield from LINAC run on 11-12 Oct 95 with the 1 mil Uranium sample.

Ythc: Scattering yield calculated from Doppler broadened ENDF cross sections using a combined resolution function consisting of channel width and burst width.

Determine the chi-square error:

$\mathrm{E} 1_{2075}=36.01138541$
$\mathrm{E} 1_{2165}=37.36220488$

Chi2 total $:=\frac{\sum_{h=2075}^{2165}\left[\frac{\left[\mathrm{TB}_{\text {total }_{\mathrm{b}}}-\mathrm{Yexp}\left(\left(\mathrm{El}_{\mathrm{h}}\right)\right)\right]}{\sigma \mathrm{Yld}}\right]_{\mathrm{h}}^{2}}{88}$
E1: Energy for the calculation

$\sigma$ Yld: Error in the experimental yield

Chi $2_{\text {total }}=2.98618337$ 
Bounce target data: Resolution fit to 1 mil Uranium sample at $66 \mathrm{eV}$ Resonance

\section{SCATTER DATA}

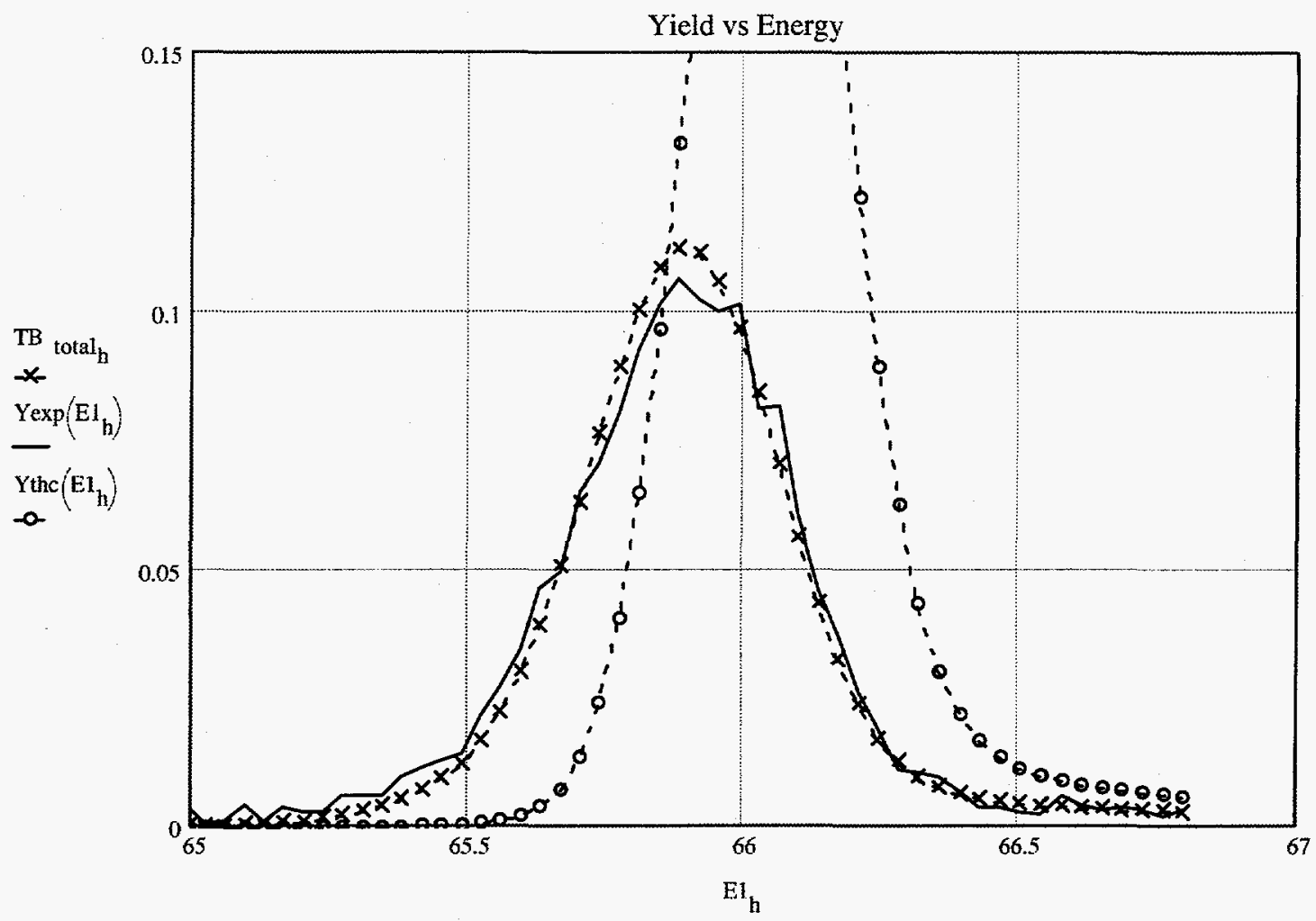

TBtotal: Scattering yield calculated from Doppler broadened ENDF cross sections using a combined resolution function consisting of channel width, burst width and detector functions. Detector efficiency used is 0.589 .

Yexp: Experimental scattering yield from LINAC run on 11-12 Oct 95 with the 1 mil Uranium sample.

Ythc: Scattering yield calculated from Doppler broadened ENDF cross sections using a combined resolution function consisting of channel width and burst width.

Determine the chi-square error:

$\mathrm{E}_{3336}=64.98989497$
$\mathrm{E}_{3386}=66.79649977$

$\mathrm{Chi} 2_{\text {total }}:=\frac{\sum_{h=3336}^{3386}\left[\frac{\left.\left.\left[\mathrm{TB} \text { total }_{\mathrm{h}}-\operatorname{Yexp}\left(\mathrm{El}_{\mathrm{h}}\right)\right)\right]\right]^{2}}{\sigma \mathrm{Yld} h}\right]_{\mathrm{h}}^{2}}{48}$

Chi2 $2_{\text {total }}=2.33736117$
E1: Energy for the calculation

oYld: Error in the experimental yield 
Bounce target data: Resolution fit to 1 mil Uranium sample at $102 \mathrm{eV}$ Resonance

\section{SCATTER DATA}

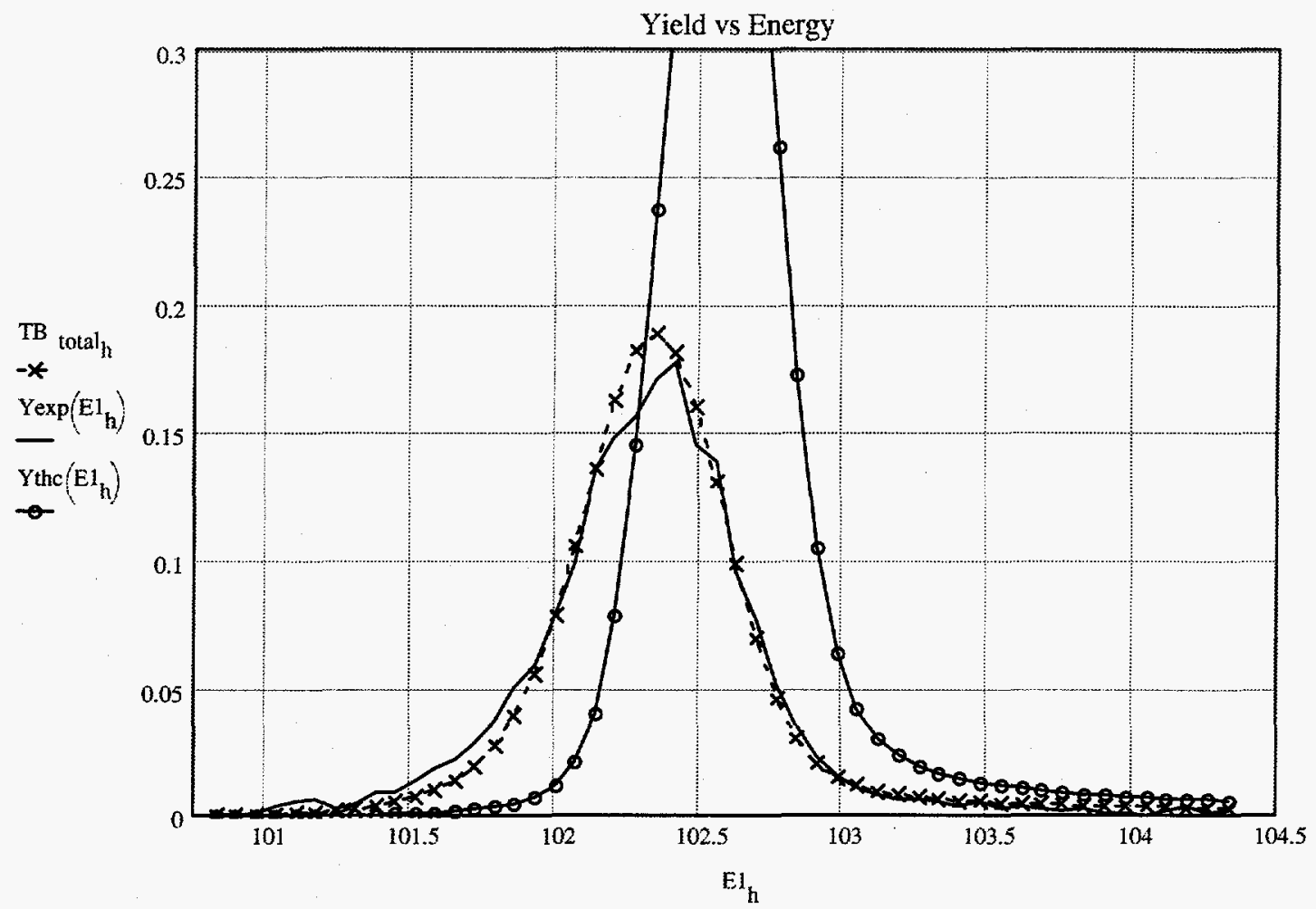

TBtotal: Scattering yield calculated from Doppler broadened ENDF cross sections using a combined resolution function consisting of channel width, burst width and detector functions. Detector efficiency used is 0.564 .

Yexp: Experimental scattering yield from LINAC run on 11-12 Oct 95 with the 1 mil Uranium sample.

Ythc: Scattering yield calculated from Doppler broadened ENDF cross sections using a combined resolution function consisting of channel width and burst width.

Determine the chi-square error:

$$
\begin{aligned}
& \mathrm{E} 1_{4060}=100.82906834 \\
& \mathrm{E} 1_{4110}=104.3381339 \\
& \sum_{h=4060}^{4110}\left[\frac{\left[{ }^{\mathrm{TB}} \text { total }_{h}-\mathrm{Yexp}\left(\left(\mathrm{E} 1_{h}\right)\right)\right]}{\sigma \mathrm{Yld}}\right]_{\mathrm{h}}^{2} \\
& \text { E1: Energy for the calculation } \\
& \sigma Y l d: \text { Error in the experimental yield } \\
& \text { Chi2 }{ }_{\text {total }}:=\frac{h=4060 L}{48} \\
& \text { Chi } 2_{\text {total }}=6.36303562
\end{aligned}
$$


Bounce target data: Resolution fit to 1 mil Uranium sample at $187 \mathrm{eV}$ Resonance

\section{SCATTER DATA}

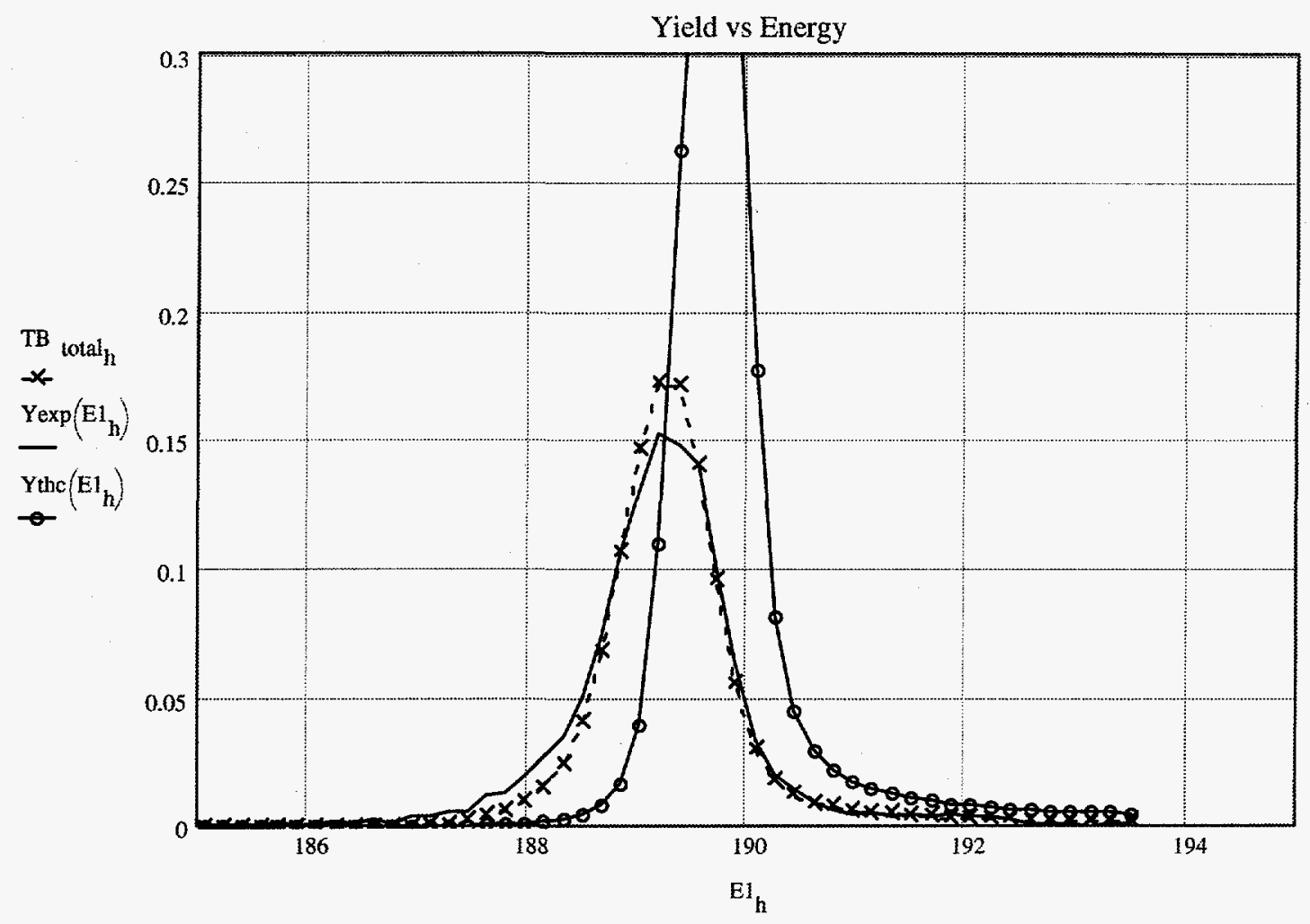

TBtotal: Scattering yield calculated from Doppler broadened ENDF cross sections using a combined resolution function consisting of channel width, burst width and detector functions. Detector efficiency used is 0.56 .

Yexp: Experimental scattering yield from LINAC run on 11-12 Oct 95 with the 1 mil Uranium sample.

Ythc: Scattering yield calculated from Doppler broadened ENDF cross sections using a combined resolution function consisting of channel width and burst width.

Determine the chi-square error:

$\mathrm{E} 1_{4830}=184.71672219$
$\mathrm{E} 1_{4880}=193.49824494$

Chi2 total $_{:=}=\frac{\sum_{\mathrm{h}=4830}^{4880}\left[\frac{\left.\left[\mathrm{TB} \text { total }_{\mathrm{h}}-\mathrm{Yexp}\left(\mathrm{E} 1_{\mathrm{h}}\right)\right)\right]}{\sigma \mathrm{Yld}}\right]_{\mathrm{h}}^{2}}{48}$
E1: Energy for the calculation

$\sigma Y \mid d:$ Error in the experimental yield

Chi $_{\text {total }}=8.23984141$ 
Bounce target data: Resolution fit to 1 mil Uranium sample at $36 \mathrm{eV}$ Resonance SCATTER DATA

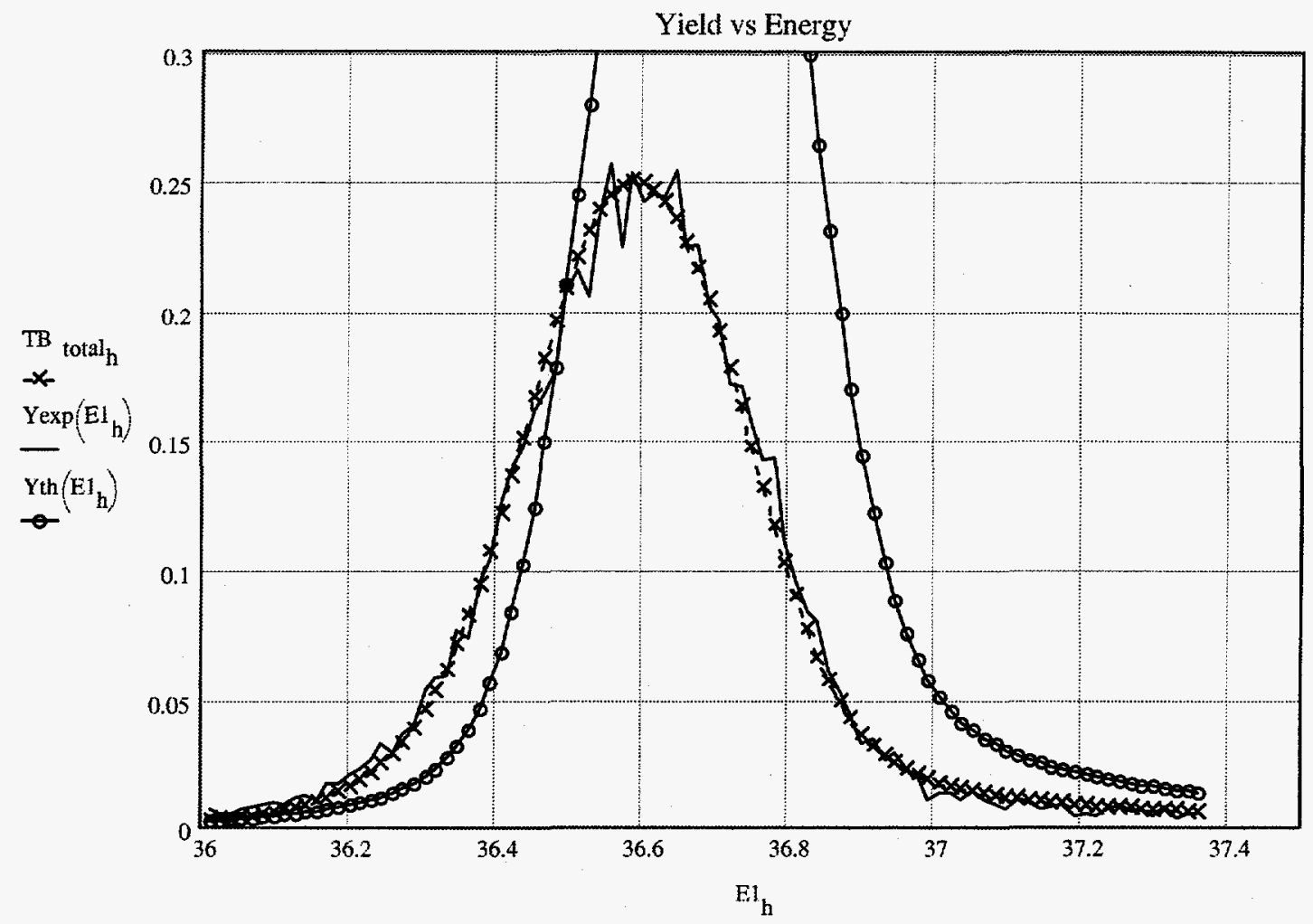

TBtotal: Scattering yield calculated from Doppler broadened ENDF cross sections using a combined resolution function consisting of all four factors. Detector efficiency used is 0.58 .

Yexp: Experimental scattering yield from LINAC run on 11-12 Oct 95 with the 1 mil Uranium sample.

Yth: $\quad$ Scattering yield calculated from Doppler broadened ENDF cross sections using a combined resolution function consisting of channel width, burst width and target functions.

Determine the chi-square error:

$\mathrm{E}_{2165}=37.362$
$\mathrm{E}_{2075}=36.011$

E1: Energy for the calculation

Chi2 total $:=\frac{\sum_{\mathrm{h}=2075}^{2165}\left[\frac{\left.\left[\mathrm{TB}_{\text {total }_{\mathrm{h}}}-\mathrm{Yexp}\left(\left(\mathrm{E} 1_{\mathrm{h}}\right)\right)\right]\right]^{2}}{\sigma \mathrm{Yld}_{\mathrm{h}}}\right]^{2}}{88}$

$\sigma$ Yld: Error in the experimental yield

Chi2 $2_{\text {total }}=1.796$ 
Bounce target data: Resolution fit to 1 mil Uranium sample at $66 \mathrm{eV}$ Resonance

\section{SCATTER DATA}

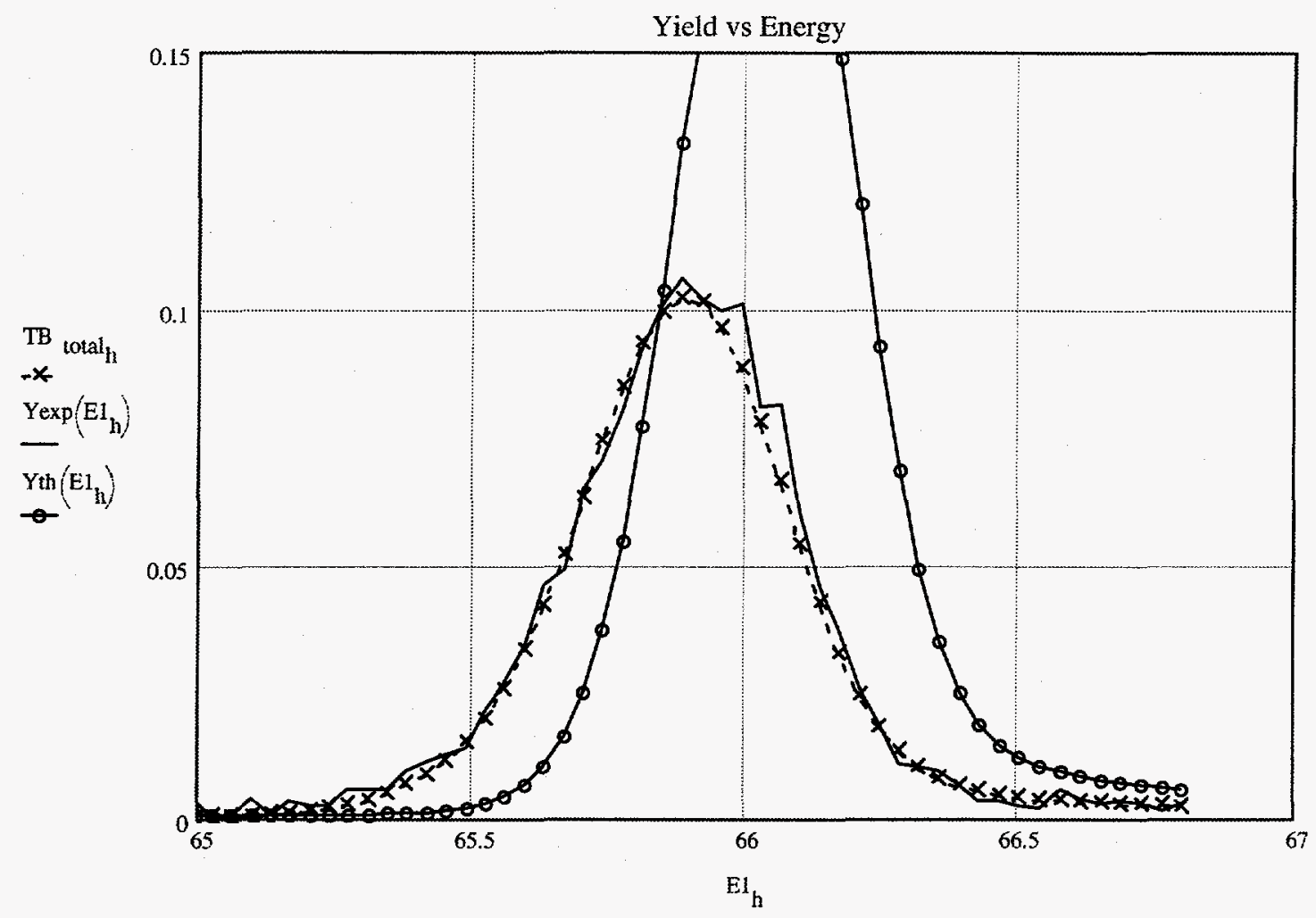

TBtotal: Scattering yield calculated from Doppler broadened ENDF cross sections using a combined resolution function consisting of all four factors. Detector efficiency used is 0.58 .

Yexp: Experimental scattering yield from LINAC run on 11-12 Oct 95 with the 1 mil Uranium sample.

Yth: Scattering yield calculated from Doppler broadened ENDF cross sections using a combined resolution function consisting of channel width, burst width and target functions.

Determine the chi-square error:

$$
\begin{aligned}
& \mathrm{E}_{3336}=64.99 \\
& \mathrm{E}_{3386}=66.796
\end{aligned}
$$

\section{E1: Energy for the calculation}

oYld: Error in the experimental yield

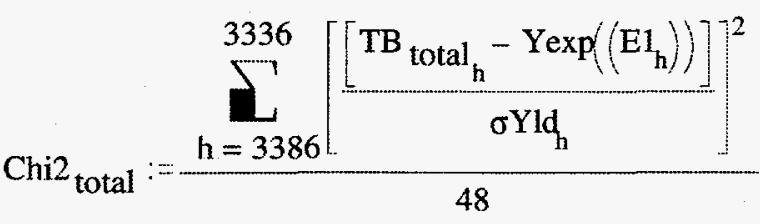

Chi $_{\text {total }}=1.384$ 
Bounce target data: Resolution fit to 1 mil Uranium sample at $102 \mathrm{eV}$ Resonance

\section{SCATTER DATA}

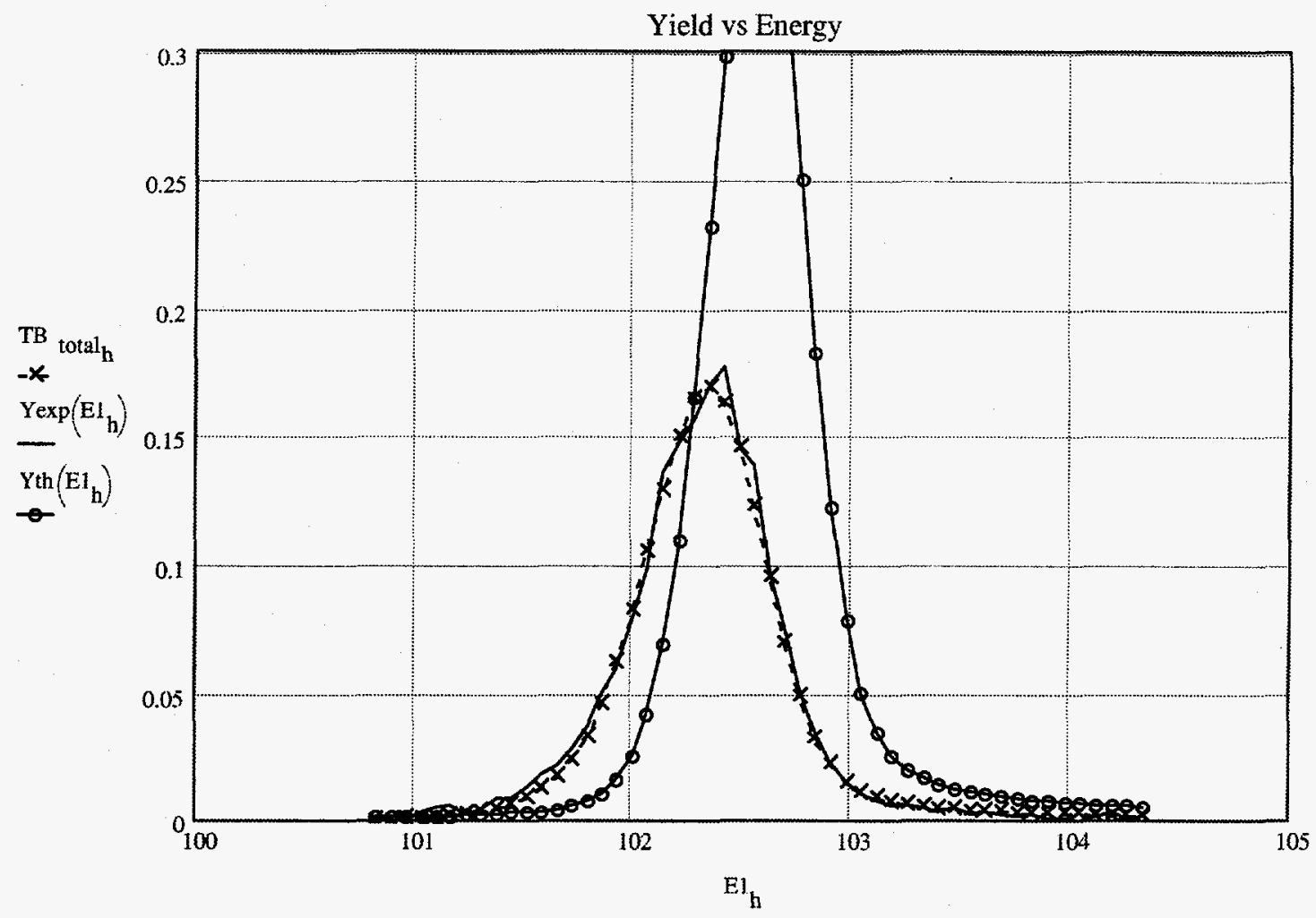

TBtotal: Scattering yield calculated from Doppler broadened ENDF cross sections using a combined resolution function consisting of all four factors. Detector efficiency used is 0.562 .

Yexp: Experimental scattering yield from LINAC run on 11-12 Oct 95 with the 1 mil Uranium sample.

Yth: $\quad$ Scattering yield calculated from Doppler broadened ENDF cross sections using a combined resolution function consisting of channel width, burst width and target functions.

Determine the chi-square error:

$\mathrm{E}_{4060}=100.829$
$\mathrm{E}_{4110}=104.338$

E1: Energy for the calculation

Chi2 total $:=\frac{\sum_{\mathrm{h}=4060}^{4110}\left[\frac{\left.\left[\mathrm{TB} \text { total }_{\mathrm{h}}-\mathrm{Yexp}\left(\left(\mathrm{E} 1_{\mathrm{h}}\right)\right)\right]\right]^{2}}{\sigma \mathrm{Yld}}\right]_{\mathrm{h}}^{2}}{48}$

oYld: Error in the experimental yield

Chi $2_{\text {total }}=2.617$ 
Bounce target data: Resolution fit to 1 mil Uranium sample at $189 \mathrm{eV}$ Resonance

\section{SCATTER DATA}

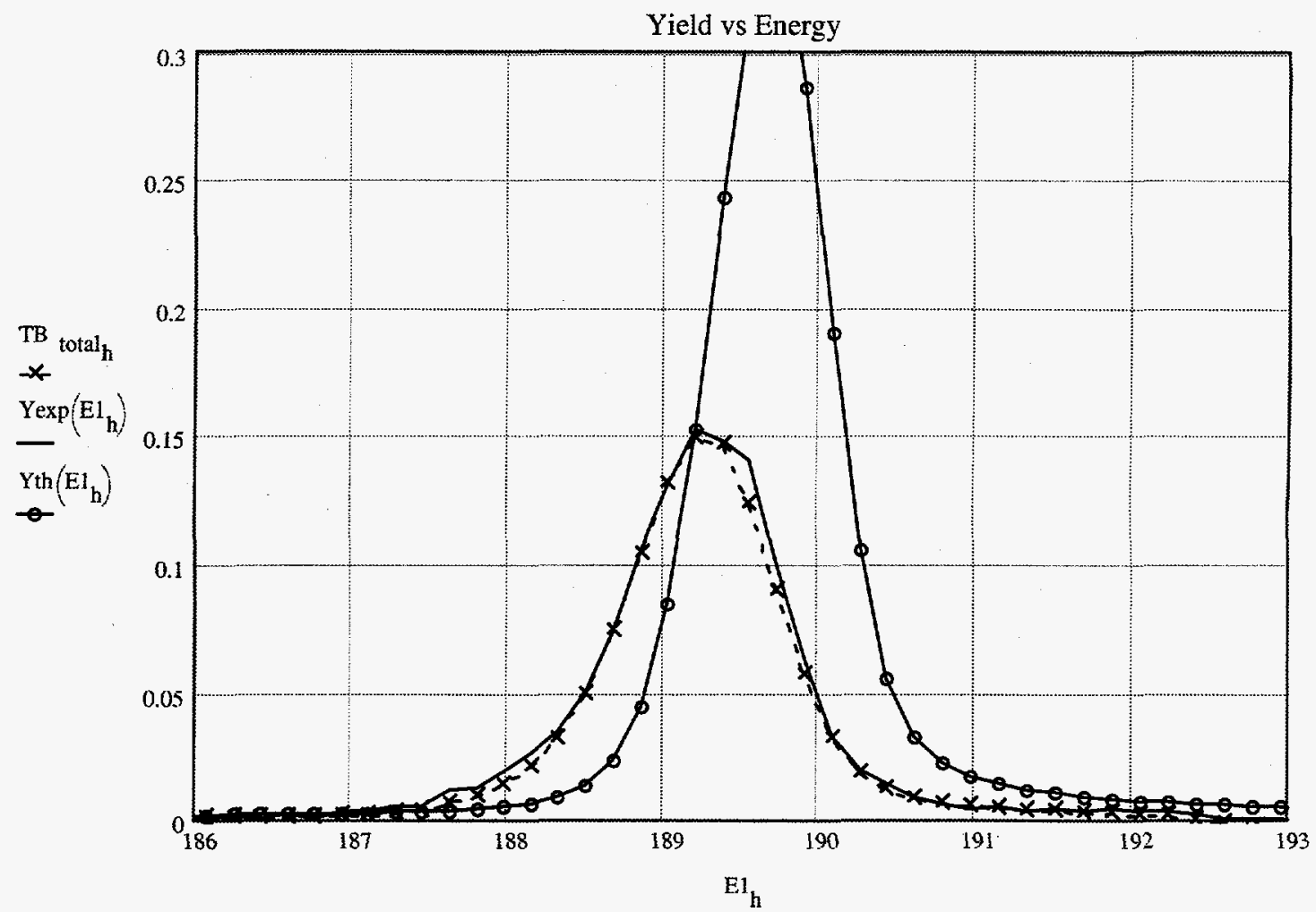

TBtotal: Scattering yield calculated from Doppler broadened ENDF cross sections using a combined resolution function consisting of all four factors. Detector efficiency used is 0.56 .

Yexp: Experimental scattering yield from LINAC run on 11-12 Oct 95 with the 1 mil Uranium sample.

Yth: Scattering yield calculated from Doppler broadened ENDF cross sections using a combined resolution function consisting of channel width, burst width and target 'functions.

Determine the chi-square error:

$$
\begin{aligned}
& \mathrm{E} 1_{4835}=185.739 \\
& \mathrm{E} 1_{4872}=192.232
\end{aligned}
$$

\section{E1: Energy for the calculation} $\sigma$ Yid: Error in the experimantal yield

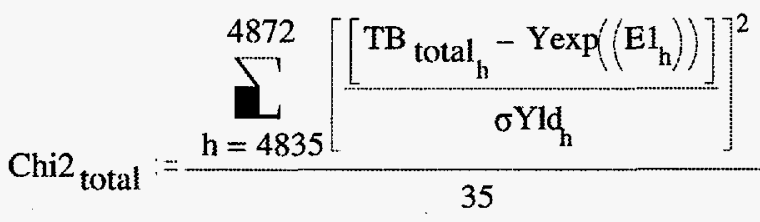

$$
\text { Chi2 } 2_{\text {total }}=2.653
$$

